\title{
CONTINUITY OF MULTIMARGINAL OPTIMAL TRANSPORT WITH REPULSIVE COST*
}

\author{
MARIA COLOMBO ${ }^{\dagger}$, SIMONE DI MARINO $^{\ddagger}$, AND FEDERICO STRA ${ }^{\S}$
}

\begin{abstract}
We provide sharp conditions for the finiteness and the continuity of multimarginal optimal transport with repulsive cost, expressed in terms of a suitable concentration property of the measure. To achieve this result, we analyze the Kantorovich potentials of the optimal plans, and we estimate the distance of any optimal plan from the regions where the cost is infinite.
\end{abstract}

Key words. multimarginal optimal transport, density functional theory, Kantorovich potential

AMS subject classifications. 35Q40, 49Q20, 49S05, 81Q05, 49K30

DOI. $10.1137 / 19 \mathrm{M} 123943 \mathrm{X}$

1. Introduction. In recent years, a new mathematical model for the strong interaction limit of the density functional theory (DFT) has been considered. For instance, in [6], Buttazzo, De Pascale, and Gori-Giorgi show that the model for the minimal interaction of $N$ electrons can be formulated in terms of a multimarginal Monge transport problem. At the same time, in [10], Cotar, Friesecke, and Klüppelberg show that an analogous optimal transportation problem describes the semiclassical limit of DFT in the case of two electrons and provides estimates from below in the general case.

In this article we prove the finiteness and continuity of multimarginal optimal transport with repulsive cost under the assumption that the measure does not concentrate too much. The article is a refinement of the results presented in [5], especially from the point of view of the assumptions, which in our work are shown to be sharp. We acknowledge also the recent preprint [2] in which the finiteness of the cost is proved in a similar fashion by dimension reduction.

To describe the problem, we fix a complete and separable (Polish) metric space $(X, \mathrm{~d})$. We consider a repulsive interaction cost given by a symmetric lower semicontinuous function $c: X \times X \rightarrow[0, \infty]$ for which there exist two nonincreasing rightcontinuous (or, equivalently, lower semicontinuous) functions $m, M:(0, \infty) \rightarrow[0, \infty)$ satisfying

$$
m\left(\mathrm{~d}\left(x_{1}, x_{2}\right)\right) \leq c\left(x_{1}, x_{2}\right) \leq M\left(\mathrm{~d}\left(x_{1}, x_{2}\right)\right) \quad \forall x_{1}, x_{2} \in X .
$$

Moreover, sometimes we will need a strong repulsion assumption, namely

$$
c(x, x)=\infty \quad \forall x \in X \quad \text { and } \quad \lim _{r \rightarrow 0^{+}} m(r)=\lim _{r \rightarrow 0^{+}} M(r)=\infty .
$$

Extending $m(0)=M(0)=\infty$ for this last case, the inequality (1.1) still holds for all $x_{1}, x_{2} \in X$. The Coulomb cost fits into this framework as $c\left(x_{1}, x_{2}\right)=\frac{1}{\left|x_{1}-x_{2}\right|}$ on $X=\mathbb{R}^{d}, d \in \mathbb{N}$, and one can take $m(r)=M(r)=\frac{1}{r}$.

\footnotetext{
${ }^{*}$ Received by the editors February 11, 2019; accepted for publication (in revised form) April 15, 2019; published electronically July 11, 2019.

https://doi.org/10.1137/19M123943X

$\dagger$ ETH, Zürich, Switzerland, and EPFL, Lausanne, Switzerland (maria.colombo@epfl.ch).

${ }_{\ddagger}^{\ddagger}$ INdAM, Scuola Normale Superiore, 56126 Pisa, Italy (simone.dimarino@altamatematica.it).

$\S$ Scuola Normale Superiore, 56126 Pisa, Italy, and Université Paris-Saclay, F-91405 Orsay, France (federico.stra@sns.it).
} 

by

For every integer $N \geq 2$, define the symmetric interaction cost $c: X^{N} \rightarrow[0, \infty]$

$$
c\left(x_{1}, \ldots, x_{N}\right):=\sum_{1 \leq i<j \leq N} c\left(x_{i}, x_{j}\right),
$$

the cost of a plan $C: \mathscr{P}\left(X^{N}\right) \rightarrow[0, \infty]$ by

$$
C(\pi):=\int_{X^{N}} c\left(x_{1}, \ldots, x_{N}\right) \mathrm{d} \pi\left(x_{1}, \ldots, x_{N}\right),
$$

and lastly, the optimal transport $\operatorname{cost} \mathscr{C}: \mathscr{P}(X) \rightarrow[0, \infty]$ associated to a marginal by

$$
\mathscr{C}(\rho):=\inf \left\{C(\pi): \pi \in \Pi_{N}(\rho)\right\}
$$

where

$$
\Pi_{N}(\rho):=\left\{\pi \in \mathscr{P}\left(X^{N}\right): P_{\#}^{i} \pi=\rho \quad \text { for } i=1, \ldots, N\right\}
$$

denotes the set of admissible transport plans and $P^{i}: X^{N} \rightarrow X$ are the projections on the $i$ th component for $i=1, \ldots, N$. The existence of a minimizer for the infimum problem in (1.4) follows from standard methods in the calculus of variations as long as $c$ is lower semicontinuous (see, for example, $[1,31,29]$ ).

Besides exploring the connection with density functional theory $[6,10,3,20]$, several authors investigated the mathematical properties of the minimizer. A natural question is whether the optimizer is induced by a map, namely if there exists a Borel map $T: X \rightarrow X$ such that $T_{\#} \rho=\rho$ (where $T_{\#} \rho$ represents the pushforward measure of the measure $\rho$ through the Borel map $T$ ) and an optimizer $\pi$ in the minimization problem in (1.4) can be represented as $\left(I d, T, T^{(2)}, \ldots, T^{(n-1)}\right)_{\#} \rho$. This question is still widely open, though some results have been obtained in $[8,7,9,30]$ regarding the possibility of approximating the cost of a minimizer with costs of these particular plans, the 1-dimensional case, and the radial case (see also the survey [15]). It is important to also mention here the negative result in [18], where they show (in the case of the repulsive harmonic cost $\left.c(x, y)=-|x-y|^{2}\right)$ an explicit density $\rho$, absolutely continuous with respect to the Lebesgue measure, such that there is a unique optimal symmetric plan, which is not induced by a map.

The asymptotic behavior as $N \rightarrow \infty$ for the Coulomb were presented in $[11,28]$ and then more precisely (up to the first order) in [25, 12].

In $[13,5]$, instead, the authors prove a duality result, which shows that the value in (1.4) can be represented via a duality argument as

$$
\sup \left\{N \int_{X} \varphi \mathrm{d} \rho: \varphi\left(x_{1}\right)+\cdots+\varphi\left(x_{N}\right) \leq c(x)\right\}
$$

the proof is carried out for the Coulomb cost but adapts to the assumption that $c$ is lower continuous; moreover, the existence of an optimal potential $\varphi$ in the dual formulation (1.5) is also proved (see also [17] for a generalization to costs not necessarily bounded from below). We remark that a general duality result has already been proven by Kellerer in [22], but the hypothesis on the cost function could not be adapted to a Coulomb-type cost.

Finally, we remark that in the context of multimarginal optimal transport problems several of the questions mentioned above are open, even with more classical cost functions such as the quadratic cost; recent developments can be found in $[16,27,24,19,15]$. 
In this paper we prove the finiteness and continuity of multimarginal optimal transport with repulsive cost under the assumption that the measure does not concentrate too much. This issue was already partly addressed in [5], where the authors present sufficient conditions for the continuity of the cost and where they analyze the Kantorovich potentials. We improve the previous results especially from the point of view of the assumptions, which in our work are shown to be sharp. The continuity of the optimal cost under the sharp conditions of the present paper is useful, for instance, in [4] to study the bond dissociation problem of certain molecules in density functional theory.

Our results depend on assumptions regarding the concentration of mass of the marginal $\rho$; therefore we introduce two quantities measuring it. Given $\mu \in \mathscr{P}(X)$, we consider the pointwise concentration of $\mu$, namely its biggest atom

$$
a(\mu):=\max _{x \in X} \mu(\{x\}),
$$

and the concentration on balls defined as

$$
\kappa(\mu, r):=\sup _{x \in X} \mu(\bar{B}(x, r)),
$$

which gives more quantitative information, since $\lim _{r \rightarrow 0} \kappa(\mu, r)=a(\mu)$. In terms of the first concentration property, we characterize the finiteness of the cost.

Theorem 1.1 (finiteness of the cost). Let $\rho \in \mathscr{P}(X)$, let $\mathscr{C}$ be the cost introduced in (1.4) under the assumptions (1.1), (1.2), and (1.3), and let $a(\rho)$ be as in (1.6). Then we have that

(i) if a $(\rho)<\frac{1}{N}$, then $\mathscr{C}(\rho)<\infty$;

(ii) if $a(\rho)>\frac{1}{N}$, then $\mathscr{C}(\rho)=\infty$;

(iii) if a $(\rho)=\frac{1}{N}$, then $\mathscr{C}(\rho)<\infty$ if and only if

$$
\int_{X \backslash\{\bar{x}\}} c(\bar{x}, x) \mathrm{d} \rho(x)<\infty \quad \forall \bar{x}: \rho(\{\bar{x}\})=\frac{1}{N} .
$$

We acknowledge here also the recent paper [2] in which the first two parts of the theorem above are proved by a slicing method and by induction on the number of atoms. Our proof is considerably shorter, and it is based instead on a dimension reduction argument, which allows us to reduce the problem to a 1-dimensional problem via a suitably chosen projection.

Next, we address in our main theorem the problem of the continuity of the cost, in which we will also use the "enlarged diagonal" for any $\alpha>0$ :

$$
D_{\alpha}=\left\{x=\left(x_{1}, \ldots, x_{N}\right) \in X^{N}: \mathrm{d}\left(x_{i}, x_{j}\right)<\alpha \quad \text { for some } i \neq j\right\} .
$$

THEOREM 1.2. Let $\mathscr{C}$ be the cost introduced in (1.4) under the assumptions (1.1), (1.2), and (1.3). Let $r>0, \delta \in(0,1 / N)$, and consider the set

$$
\mathscr{K}_{r, \delta}:=\{\rho \in \mathscr{P}(X): \kappa(\rho, r)<\delta\} .
$$

Then we have the following:

(i) $\mathscr{C}$ is Lipschitz in $\mathscr{K}_{r, \delta}$ with respect to the total variation norm on $\mathscr{P}(X)$.

(ii) If, in addition, the cost $c$ is continuous, $\mathscr{C}$ is continuous in $\{\rho \in \mathscr{P}(X)$ : $a(\rho)<1 / N\}$ with respect to the weak topology, or equivalently with respect to the Wasserstein distance $W_{1}$. 
(iii) If the cost $c$ is Lipschitz outside $D_{\alpha}$ for every $\alpha>0$, then $\mathscr{C}$ is Lipschitz in $\mathscr{K}_{r, \delta}$ with respect to the Wasserstein $W_{1}$ distance on $\mathscr{P}(X)$.

A consequence of Theorem 1.2(i) is that $\mathscr{C}$ is locally Lipschitz on $\{\rho \in \mathscr{P}(X)$ : $a(\rho)<1 / N\}$ with respect to the strong topology on $\mathscr{P}(X)$. Similarly, under the assumptions on the cost in Theorem 1.2(iii), $\mathscr{C}$ is locally Lipschitz in $\{\rho \in \mathscr{P}(X)$ : $a(\rho)<1 / N\}$ with respect to the Wasserstein distance. On the other hand, it is not true that the cost is continuous, even with the strong topology, on the set $\{\rho \in$ $\mathscr{P}(X): \mathscr{C}(\rho)<\infty\}$, even in the case of the Coulomb cost in $X=\mathbb{R}^{d}$. Indeed, it is clear that if $a(\rho)=1 / N$ for some $\rho$ of finite cost, we can enlarge the Dirac delta of size $1 / N$ in $\rho$ (reducing the mass slightly elsewhere) to obtain a sequence of measures of infinite cost which converge strongly to $\rho$. On the other side, with a little bit more work we can approximate any $\rho$ of this type with measures of finite energy creating a discontinuity in $\rho$; see Lemma 3.5 below.

The result in Theorem 1.2 is based on two key ideas. First, in Theorem 1.3 we provide quantitative bounds regarding the distance of the support from the diagonals $x_{i}=x_{j}, i \neq j$ (where the cost is infinite). Once this result is established, the minimization problem (1.4) becomes fully equivalent to the same problem with a cost truncated from above (see Lemma 5.1). Under the boundedness assumption for the cost, we can refer to more classical optimal transport results to obtain the existence of optimal potentials as well as their estimates. Since these results might have an interest which goes beyond the proof of Theorem 1.2, we describe them in the next two subsections.

1.1. Diagonal bounds. For $\alpha>0$ define the "enlarged diagonals"

$$
\begin{array}{ll}
D_{\alpha}=\left\{x=\left(x_{1}, \ldots, x_{N}\right) \in X^{N}: \mathrm{d}\left(x_{i}, x_{j}\right)<\alpha\right. & \text { for some } i \neq j\}, \\
\bar{D}_{\alpha}=\left\{x=\left(x_{1}, \ldots, x_{N}\right) \in X^{N}: \mathrm{d}\left(x_{i}, x_{j}\right) \leq \alpha\right. & \text { for some } i \neq j\} .
\end{array}
$$

Notice that in general $\bar{D}_{\alpha}$ is not the closure of $D_{\alpha}$ (which would be denoted by $\overline{D_{\alpha}}$ if needed) but rather contains it. We also introduce an enlarged diagonal which is more intrinsic in terms of the cost, rather than of the distance

$$
D^{h}=\left\{x=\left(x_{1}, \ldots, x_{N}\right) \in X^{N}: c\left(x_{i}, x_{j}\right)>h \quad \text { for some } i \neq j\right\} .
$$

The set $D^{h}$ is a more tailored version of $D_{\alpha}$, but of course we have $D^{M(\alpha)} \subseteq D_{\alpha} \subseteq$ $D^{m(\alpha)}$, and, for example, in the case $c(x, y)=f(d(x, y))$ they coincide up to a composition with $f$ or its inverse.

We can provide some diagonal bounds for the optimal plan, improving the corresponding result in [5, Theorem 2.4]. Notice that in this theorem we do not require the strong repulsion assumption (1.2); this is important since in the crucial Lemma 5.1 we in fact apply it to a bounded cost.

Theorem 1.3 (diagonal bounds). Let $\rho \in \mathscr{P}(X), r>0$; let us consider an optimal plan $\pi \in \Pi(\rho)$ in (1.4) under the assumptions (1.1) and (1.3), and let $\kappa(\rho, r)$ be as in (1.6). Then we have the following:

(i) If $\kappa(\rho, r)<\frac{1}{2(N-1)}$ and $h>2(N-1) M(r)$, then $\pi\left(D^{h}\right)=0$.

(ii) If $\kappa(\rho, r)<\frac{1}{N}$ whenever we have $h, \beta>0$ satisfying

$$
h>2(N-1) M(\beta / 2), \quad m(\beta)>\frac{\mathscr{C}(\rho)}{1-N \kappa(\rho, r)}, \quad \beta / 2 \leq r,
$$


then we have $\pi\left(D^{h}\right)=0$. Moreover, under assumption (1.2), there always exist such $h, \beta>0$, and they can be also chosen depending only on $r, N$, and $\delta:=1-N \kappa(\rho, r)$.

In particular, in both cases we have $\pi\left(D_{\alpha}\right)=0$ whenever $m(\alpha) \geq h$.

Notice that, while in (ii) the assumption on the measure is sharp, the behavior of the estimate of $h$ with respect to $N$ and $r$ is not very nice. Indeed, it depends on $\mathscr{C}(\rho)$, which can be avoided by using Theorem 4.1, at the cost of losing a factor $\left(\begin{array}{c}N \\ 2\end{array}\right)$. This is why we also kept estimate (i), which is sharper in the behavior on $N$ and $r$ despite not being optimal on the assumption.

1.2. Kantorovich potentials. We recall here the existence of optimal potentials, which is the main result of [13]. In the paper the proof is written for the Coulomb cost and for probabilities $\rho$ with no atoms. Using some ideas present in [5], we provide a sharper version that works for every $\rho$ such that $a(\rho)<1 / N$.

TheOREM 1.4. Let $\mathscr{C}$ be the cost introduced in (1.4) under the assumptions (1.1), (1.2), and (1.3), and let $\rho \in \mathscr{P}(X)$ with $a(\rho)<1 / N$. Then the duality formula

$$
\mathscr{C}(\rho)=\sup \left\{N \int_{X} \varphi \mathrm{d} \rho: \varphi\left(x_{1}\right)+\cdots+\varphi\left(x_{N}\right) \leq c(x)\right\}
$$

holds, and the supremum in the right-hand side is realized by a potential $\varphi \in L^{1} \cap$ $L^{\infty}(\rho)$.

Proof. We deduce this theorem since by Lemma 2.1 we have $\rho \in \mathscr{K}_{r, \delta}$ for some $r>0$ and $\delta<1 / N$; then we use Lemma 5.1, which in turn uses the existence of optimal potentials for bounded costs, which was proved in [22].

To prove the continuity of the cost in Theorem 1.2 , we need to obtain uniform estimates on these potentials when $\rho$ varies in a set $\mathscr{K}_{r, \delta}$. Notice that in the following theorem we do not assume the strong repulsion assumption (1.2).

Proposition 1.5 ( $L^{\infty}$ and Lip bounds on the Kantorovich potential). Let $\mathscr{C}$ be the cost introduced in (1.4) under the assumptions (1.1) and (1.3). Let $r>0$, $\delta \in(0,1 / N)$, and consider the set $\mathscr{K}_{r, \delta}$ introduced in (1.8).

Then there exists a function $h:=h(r, \delta)$ (given, for example, by (5.3)) such that the following hold:

(i) There exist Kantorovich potentials $\varphi_{\rho}$ which are uniformly bounded in $\mathscr{K}_{r, \delta}$ :

$$
\sup _{\rho \in \mathscr{K}_{r, \delta}}\left\|\varphi_{\rho}\right\|_{L^{\infty}(X)}<\left(\begin{array}{c}
N \\
2
\end{array}\right) \cdot h(r, \delta) .
$$

(ii) If the cost c is Lipschitz outside $D_{\alpha}$ for every $\alpha>0$, then there exist Kantorovich potentials $\varphi_{\rho}$ which are uniformly Lipschitz in $\mathscr{K}_{r, \delta}$ :

$$
\sup _{\rho \in \mathscr{K}_{r, \delta}}\left\|\varphi_{\rho}\right\|_{\operatorname{Lip}(X)}<(N-1) \cdot\left\|c^{h(r, \delta)}\right\|_{\operatorname{Lip}(X)}
$$

where $c^{h}$ is defined as in (5.1).

1.3. Examples. We summarize here three particular examples that fall inside the setting of the previous theorems.

Coulomb in $\mathbb{R}^{d}$. The model case is the Coulomb interaction in $\mathbb{R}^{3}$. This is how the problem originated in the context of DFT. The ambient space is $\mathbb{R}^{d}$, and the cost $c(x, y)=1 /|x-y|$. In this case we have $m(t)=M(t)=\frac{1}{t}$. Since this is maybe the most interesting example, we provide specific and quantitative estimates for every theorem in section 6 . 
Case $c=\phi \circ \mathrm{d}$. A specific instance of this kind would be a cost of the form $c\left(x_{1}, x_{2}\right)=\phi\left(\mathrm{d}\left(x_{1}, x_{2}\right)\right)$, where $\phi:[0, \infty) \rightarrow[0, \infty]$ is a lower semicontinuous function such that

- $\phi(0)=\infty$, and hence $\lim _{r \rightarrow 0^{+}} \phi(r)=\infty$, and

- $\left.\phi\right|_{[r, \infty)}$ is bounded for every $r>0$.

In this case, $m$ and $M$ could be given by

$$
m(r):=\min _{r^{\prime} \in[0, r]} \phi\left(r^{\prime}\right), \quad M(r):=\sup _{r^{\prime} \in[r, \infty)} \phi\left(r^{\prime}\right) .
$$

From the definition it follows that $m$ and $M$ are nonincreasing and right-continuous, $m(r) \leq \phi(r) \leq M(r)$, and $\lim _{r \rightarrow 0^{+}} m(r)=\infty$. We define also the pseudoinverse $m^{-1}:[0, \infty) \rightarrow(0, \infty]$ by

$$
m^{-1}(t):=\max \{r \in(0, \infty]: m(r) \geq t\} .
$$

Then $m^{-1}$ is nonincreasing and left-continuous and satisfies the important relation $m\left(m^{-1}(t)\right) \geq t$.

Green function of $\Delta$. Noticing that the potential $1 /|x-y|$ is the fundamental solution of the Laplacian in $\mathbb{R}^{3}$, the first case can be generalized to a Riemannian manifold $M$ where the cost is given by $c(x, y)=G(x, y)$, the fundamental solution of $\Delta_{x} G(x, y)=\delta_{y}$. If the manifold is compact, then it is clear that $c$ satisfies the previous hypotheses, but they could be verified also on some noncompact manifolds, like they are in $\mathbb{R}^{d}$ because of the translation invariance.

2. Preliminary results. For all the functions introduced so far, sometimes we will drop the $N$ dependence whenever it will be clear from the context.

We will use the notation $P^{i}: X^{N} \rightarrow X$ to denote the projection on the $i$ th coordinate and also $P^{i_{1}, \ldots, i_{k}}: X^{N} \rightarrow X^{k}$ to denote the projection on the coordinates $i_{1}, \ldots, i_{k}$. Moreover, given $\pi \in \mathscr{P}\left(X^{N}\right)$ we denote $P_{\text {sym }}(\pi)=\frac{1}{N !} \sum_{\sigma \in S_{N}}\left(P^{\sigma(1), \ldots, \sigma(N)}\right)_{\#} \pi$ and notice that $C(\pi)=C\left(P_{\text {sym }}(\pi)\right)$ thanks to the symmetry of the cost. Here $S_{N}$ denotes the group of all permutations of $\{1, \ldots, N\}$.

2.1. Properties of the concentration. Clearly the uniform concentration condition measured by $\kappa$ is stronger than the pointwise one encoded by $a$. However, thanks to a compactness argument, the next lemma shows that the two are in fact almost equivalent.

Lemma 2.1. Let $\rho \in \mathscr{P}(X)$, and assume that $a(\rho)<\delta$. Then there exists $r>0$ such that $\kappa(\rho, r)<\delta$.

Proof. Fix $\delta^{\prime}$ such that $a(\rho)<\delta^{\prime}<\delta$. Since $\rho$ is tight, we can find a compact subset $K \subset X$ such that $\rho\left(K^{c}\right)<\delta^{\prime}$. Given $x \in X$, one has $\lim _{r \rightarrow 0^{+}} \rho(\bar{B}(x, r))=$ $\rho(\{x\}) \leq a(\rho)<\delta^{\prime}$; therefore, for every $x$ there exists a positive radius $r_{x}$ such that

$$
\rho\left(\bar{B}\left(x, 3 r_{x}\right)\right)<\delta^{\prime} .
$$

Since $K$ is compact, we can find a finite number of points $x_{1}, \ldots, x_{k}$ such that $K \subset$ $\bigcup_{i=1}^{k} \bar{B}\left(x_{i}, r_{x_{i}}\right)$. Let $r=\min \left\{r_{x_{1}}, \ldots, r_{x_{k}}\right\}$. If $\mathrm{d}(x, K)>r$, then $\bar{B}(x, r) \subset K^{c}$; hence $\rho(\bar{B}(x, r))<\delta^{\prime}$. If $\mathrm{d}(x, K) \leq r$, then $\mathrm{d}\left(x, x_{i}\right) \leq r+r_{x_{i}} \leq 2 r_{x_{i}}$ for some $i=1, \ldots, k$; therefore, $\bar{B}(x, r) \subset \bar{B}\left(x_{i}, 3 r_{x_{i}}\right)$, and hence $\rho(\bar{B}(x, r))<\delta^{\prime}$. This implies $\kappa(\rho, r) \leq \delta^{\prime}<\delta$.

Lemma 2.2. Assume that $\rho, \eta \in \mathscr{P}(X)$. Then for every $r, r^{\prime}>0$ we have

$$
\left(r-r^{\prime}\right) \cdot\left(\kappa\left(\eta, r^{\prime}\right)-\kappa(\rho, r)\right) \leq W_{1}(\rho, \eta) .
$$


Proof. By symmetry we can assume $r>r^{\prime}$. For any $\varepsilon>0$ small, let us take $x \in X$ such that $\eta\left(B\left(x, r^{\prime}\right)\right) \geq \kappa\left(\eta, r^{\prime}\right)-\varepsilon$. Then we can consider an optimal plan $\gamma$ between $\eta$ and $\rho$, and let $A=B\left(x, r^{\prime}\right) \times(X \backslash B(x, r))$; we have that

$$
\begin{aligned}
\gamma(A) & =\gamma\left(B\left(x, r^{\prime}\right) \times X\right)-\gamma\left(B\left(x, r^{\prime}\right) \times B(x, r)\right) \\
& \geq \gamma\left(B\left(x, r^{\prime}\right) \times X\right)-\gamma(X \times B(x, r)) \\
& =\eta\left(B\left(x, r^{\prime}\right)\right)-\rho(B(x, r)) \geq \kappa\left(\eta, r^{\prime}\right)-\varepsilon-\kappa(\rho, r) .
\end{aligned}
$$

Hence we can estimate

$$
W_{1}(\rho, \eta) \geq \int_{A} d(x, y) \mathrm{d} \gamma(x, y) \geq\left(r-r^{\prime}\right) \gamma(A) \geq\left(r-r^{\prime}\right) \cdot\left(\kappa\left(\eta, r^{\prime}\right)-\varepsilon-\kappa(\rho, r)\right),
$$

and we conclude by the arbitrariness of $\varepsilon$.

Lemma 2.3. Assume that $\rho \in \mathscr{P}(X)$ satisfies $\kappa(\rho, r)<\delta$ for some $r>0$, and let $\rho_{n} \rightarrow \rho$. Then for every $r^{\prime} \in(0, r)$ one has $\kappa\left(\rho_{n}, r^{\prime}\right)<\delta$ for $n$ large enough.

In particular, if $a(\rho)<\delta$, then $a\left(\rho_{n}\right)<\delta$ definitely in $n$.

Proof. We can assume that the distance is bounded, considering the modified distance $d_{M}(x, y)=\min \{M, d(x, y)\}$, for $M$ big enough. If the distance is bounded, we have that $\rho_{n} \rightarrow \rho$ if and only if $W_{1}\left(\rho_{n}, \rho\right) \rightarrow 0$. But then for $r^{\prime}<r$ we can apply Lemma 2.2 in order to get

$$
\limsup _{n \rightarrow \infty} \kappa\left(\rho_{n}, r^{\prime}\right) \leq \limsup _{n \rightarrow \infty}\left\{\kappa(\rho, r)+\frac{W_{1}\left(\rho_{n}, \rho\right)}{r-r^{\prime}}\right\}=\kappa(\rho, r)<\delta .
$$

Proposition 2.4 (good projection). Let $\rho \in \mathscr{P}(X)$ with $a(\rho)<\delta$. Then there exists $P \in \operatorname{Lip}_{1}(X)$ such that $a\left(P_{\#} \rho\right)<\delta$. Such a $P$ will be called a good projection.

Proof. We start from the case where $X$ is a finite-dimensional normed vector space, i.e., $X \simeq \mathbb{R}^{d}$. It is sufficient to show that there exists $P_{d} \in \operatorname{Lip}\left(\mathbb{R}^{d} ; \mathbb{R}^{d-1}\right)$ such that $a\left(P_{d \#} \rho\right)<\delta$. Then we conclude by taking $P=P_{2} \circ \cdots \circ P_{d}$. The statement is true if we are able to find a direction $v \in \mathbb{R}^{d}$ such that $\rho(l)<\delta$ for every line $l$ parallel to $v$. In fact, then we can write $\mathbb{R}^{d} \simeq \mathbb{R}^{d-1} \oplus\langle v\rangle$ and take $P_{d}$ to be the projection onto the first factor. Fix a positive $\varepsilon<[\delta-a(\rho)] / 2$. Let $\left\{x_{i}\right\}_{i}$ be the at most countable set of atoms of $\rho$. Take out a finite number of them, $x_{1}, \ldots, x_{n}$, such that the mass of the remaining ones is small, namely

$$
\sum_{i>n} \rho\left(\left\{x_{i}\right\}\right)<\varepsilon
$$

The directions $v_{i j}=x_{i}-x_{j}$ are forbidden. Consider the nonatomic measure

$$
\tilde{\rho}=\rho-\sum_{i \geq 1} \rho\left(\left\{x_{i}\right\}\right) \delta_{x_{i}} .
$$

This measure is additive on finite unions of distinct lines, because the intersections are finite sets of points, which have zero measure with respect to $\tilde{\rho}$. Therefore, there is only a finite number of lines $l_{1}, \ldots, l_{k}$ with $\tilde{\rho}\left(l_{i}\right) \geq \varepsilon$. Let $v_{i}$ denote a direction parallel to $l_{i}$. This procedure rules out another finite number of directions, $v_{1}, \ldots, v_{k}$. Now take a direction $v$ which is not parallel to any of the $v_{i j}$ or $v_{i}$. If $l$ is a line parallel 
to $v, l$ can contain at most one of the points $x_{1}, \ldots, x_{n}$ (otherwise $v$ would be parallel to some $v_{i j}$ ) and $\tilde{\rho}(l)<\varepsilon$ (otherwise $v$ would be parallel to some $v_{i}$ ). Therefore,

$$
\rho(l) \leq \tilde{\rho}(l)+\max _{i=1, \ldots, n} \rho\left(\left\{x_{i}\right\}\right)+\sum_{i>n} \rho\left(\left\{x_{i}\right\}\right)<\varepsilon+a(\rho)+\varepsilon<\delta .
$$

Assume now that $X=\ell^{\infty}$ and $\rho \in \mathscr{P}\left(\ell^{\infty}\right)$ is tight. It is well known (see, for instance, [26, Lemma 5.7]) that $\ell^{\infty}$ has the metric approximation property; that is, for every compact set $K \subset \ell^{\infty}$ and every $\varepsilon>0$ there is a linear operator $T: \ell^{\infty} \rightarrow \ell^{\infty}$ of finite rank with operator norm $\|T\| \leq 1$ and $\sup _{x \in K}\|T x-x\|_{\infty} \leq \varepsilon$. Since $\rho$ is tight, there are increasing compact sets $K_{n}$ such that $\rho\left(K_{n}^{c}\right)<1 / n$. $\rho$ is clearly concentrated on the set $H=\bigcup_{n} K_{n}$. Let $T_{n}: \ell^{\infty} \rightarrow \ell^{\infty}$ be a finite-rank linear operator with $\left\|T_{n}\right\| \leq 1$ and $\sup _{x \in K_{n}}\left\|T_{n} x-x\right\|_{\infty} \leq 1 / n$. For every $x \in H$ we have $T_{n} x \rightarrow x$ as $n \rightarrow \infty$; therefore, $T_{n \#} \rho \rightarrow \rho .{ }^{1}$ But then, by Lemma 2.3, $a\left(T_{n \#} \rho\right)<\delta$ for $n$ sufficiently large. The measure $T_{n \#} \rho$ is supported on a finite-dimensional vector subspace of $\ell^{\infty}$ (the image of $T_{n}$ ); therefore, we already know that there is a good projection $Q$ for it. A good projection for $\rho$ itself is then given by $P=Q \circ T_{n}$.

In the general case of a Polish space $(X, \mathrm{~d})$, we simply need to embed it isometrically, $\iota: X \rightarrow \ell^{\infty}$, by means of $\iota(x)=\left(\varphi_{n}(x)\right)_{n}$, where $\varphi_{n}(x)=\mathrm{d}\left(x, x_{n}\right)-\mathrm{d}\left(x, x_{0}\right)$ and $\left\{x_{n}\right\}_{n} \subset X$ is a countable dense set. By the Ulam lemma, $\rho$ is tight, and so is $\iota_{\#} \rho \in \mathscr{P}\left(\ell^{\infty}\right)$. Clearly $a\left(\iota_{\#} \rho\right)=a(\rho)<\delta$; therefore, we can find a good projection $Q$ for $\iota_{\#} \rho$ and a good projection for $\rho$ is given by $P=Q \circ \iota$.

Remark 2.5. The previous proposition remains true when $\rho$ is a tight finite nonnegative measure on a generic metric space $X$. The only modification is to observe that we just need to embed only $\operatorname{supp}(\rho) \hookrightarrow \ell^{\infty}$, which is $\sigma$-compact and closed and thus Polish.

Proposition 2.4 will be used to prove the finiteness of the cost under the assumption that $a(\rho)<1 / N$. To deal with the other concentration condition $\kappa(\rho, r)<1 / N$, one could hope to extend the good projection in the following way. However, we have not been able to establish the truth of the next conjecture; therefore, we had to find another way to get the bound of the cost (see Theorem 4.1). The conjecture, however, seems interesting enough from the measure theoretic perspective, so we state it anyway.

Conjecture 2.6 (good projection, quantitative version). Let $\rho \in \mathscr{P}\left(\mathbb{R}^{d}\right)$ with $\kappa(\rho, r)<\delta$. Then for every $\varepsilon>0$ there exists $P \in \operatorname{Lip}_{1}\left(\mathbb{R}^{d}\right)$ such that $\kappa\left(P_{\#} \rho, r^{\prime}\right)<$ $\delta+\varepsilon$ for some $r^{\prime}(r, d, \delta, \varepsilon)>0$.

\section{Characterization of finiteness of the cost.}

LEMma 3.1 (monotone plan). Let $\rho \in \mathscr{P}(\mathbb{R})$. Then there exists $\pi_{\rho} \in \Pi_{N}(\rho)$ such that for $\pi_{\rho}$-a.e. $x \in \mathbb{R}^{N}$ and for every $i \neq j$ we have $\rho\left(\left[x_{i}, x_{j}\right]\right) \geq \frac{1}{N}$. In particular, if $\kappa(\rho, r)<\frac{1}{N}$, then $\pi_{\rho}\left(D_{2 r}\right)=0$.

Remark 3.2. In the following proof we will consider $\pi_{\rho}$ as the unique symmetric monotone plan (which is a plan with the property that every $x, y$ in its support are well ordered, as defined in [7]). Since we are only interested in its final properties, we will not prove that it is monotone (even if it is obvious), nor will we discuss its uniqueness; a proof of these properties in the case of atomless $\rho$ can be found in [7].

\footnotetext{
${ }^{1}$ Indeed, if $f \in C_{b}\left(\ell^{\infty}\right)$, one has $\int f \mathrm{~d} T_{n \#} \rho=\int f \circ T_{n} \mathrm{~d} \rho \rightarrow \int f \mathrm{~d} \rho$ by dominated convergence.
} 
Proof. Let us consider $F:[0,1] \rightarrow \mathbb{R}$ defined as $F(t)=\sup \{x: \rho((-\infty, x])<t\}$. Then let us define

$$
\pi_{\rho}=N \cdot P_{\text {sym }}\left(\left.\left(F(t), F\left(t+\frac{1}{N}\right), \ldots, F\left(t+\frac{N-1}{N}\right)\right)_{\#} \mathcal{L}\right|_{\left[0, \frac{1}{N}\right]}\right) .
$$

We claim that $\pi_{\rho}$ is a plan with the properties we want.

It is clear to see that $F$ is a pseudoinverse of the cumulative distribution function of $\rho$. In particular, we have $F_{\#} \mathcal{L}_{[0,1]}=\rho$, and so we obtain that $\pi_{\rho} \in \Pi_{N}(\rho)$. Moreover, from the definition of $F$ we deduce

$$
\rho(-\infty, F(t)) \leq t \leq \rho((-\infty, F(t)]) .
$$

In particular, we get immediately $\rho\left(\left[F\left(t+\frac{i}{N}\right), F\left(t+\frac{j}{N}\right)\right]\right) \geq \frac{|i-j|}{N}$, which implies the wanted property on $\pi_{\rho}$.

Now, if we add the hypothesis that $\kappa(\rho, r)<\frac{1}{N}$, we have that $\rho\left(\left[x_{i}, x_{j}\right]\right) \geq \frac{1}{N}$ implies $\left|x_{i}-x_{j}\right| \geq 2 r$. In fact, if this were not the case, then

$$
\frac{1}{N} \leq \rho\left(\left[x_{i}, x_{j}\right]\right) \leq \rho\left(B\left(\frac{x_{i}+x_{j}}{2}, r\right)\right) \leq \kappa(\rho, r)<\frac{1}{N} .
$$

But then $D_{2 r} \subseteq \bigcup_{i \neq j}\left\{x: \rho\left(\left[x_{i}, x_{j}\right]\right)<\frac{1}{N}\right\}$, and since we know that the second set is $\pi_{\rho}$-null, we have also that $\pi_{\rho}\left(D_{2 r}\right)=0$.

We present here a simple proof of the finiteness of the cost depending on the existence of good projections, before moving on to the more powerful, but maybe less intuitive, Theorem 4.1. This result appears also in the recent preprint [2, Theorem 1.1], where it is proved in a longer way using at the core a dimension reduction argument similar to our good projection, but working always in the original ambient space and therefore not fully exploiting the simpler structure of the 1-dimensional problem.

Proof of Theorem 1.1(i). We claim that, given $\rho \in \mathscr{P}(X)$ such that $a(\rho)<1 / N$, there exists a plan $\pi \in \Pi(\rho)$ such that $\pi\left(D_{\alpha}\right)=0$ for some $\alpha>0$. In particular, the statement follows since

$$
\mathscr{C}(\rho) \leq C(\pi) \leq\left(\begin{array}{c}
N \\
2
\end{array}\right) M(\alpha)<\infty
$$

To show the claim, take a good projection $P \in \operatorname{Lip}_{1}(X)$ given by Proposition 2.4, and consider the measure $\nu=P_{\#} \rho$; in particular, we have $a(\nu)<\frac{1}{N}$ and, thanks to Lemma 2.1, also that $\kappa(\nu, r)<\frac{1}{N}$ for some $r>0$. By the disintegration theorem there are probabilities $\rho_{t} \in \mathscr{P}(X)$ such that $\rho=\rho_{t} \otimes \nu(t)$ and $t \mapsto \rho_{t}$ is Borel (see, for example, [14, section III-70]).

Let $\tilde{\pi} \in \Pi(\nu)$ be a plan given by Lemma 3.1, and let $\pi \in \Pi(\rho)$ be any plan such that $(P, \ldots, P)_{\#} \pi=\tilde{\pi}$. Such a plan can be built by arbitrarily mapping the measures $\rho_{t}$ onto one another. In particular, we will have that for every $\left(x_{1}, \ldots, x_{N}\right) \in \operatorname{supp}(\pi)$ we have $\left(P\left(x_{1}\right), \ldots, P\left(x_{n}\right)\right) \in \operatorname{supp}(\tilde{\pi})$, and so we get that $\pi\left(D_{\alpha}\right)=0$ as long as $\tilde{\pi}\left(D_{\alpha}\right)=0$, thanks to the fact that $P$ is 1-Lipschitz.

Since we have $\kappa(\nu, r)<\frac{1}{N}$, Lemma 3.1 gives that $\tilde{\pi}\left(D_{2 r}\right)=0$, and so we can conclude $\pi\left(D_{2 r}\right)=0$.

Proof of Theorem 1.1(ii). We prove that every $\rho \in \mathscr{P}(X)$ such that $\mathscr{C}(\rho)<\infty$ satisfies $a(\rho) \leq 1 / N$. Let $\pi \in \Pi(\rho)$ be an optimal plan. Since $\mathscr{C}(\rho)=C(\pi)<\infty$, we 
infer that $\pi(D)=0$. Let $\bar{x} \in \arg \max \{\rho(\{x\}): x \in X\}$, so that $\rho(\{\bar{x}\})=a(\rho)$, and define $X_{*}=\{\bar{x}\}^{c}$. For every $i=1, \ldots, N$ one has

$$
\rho(\{\bar{x}\})=P_{\#}^{i} \pi(\{\bar{x}\})=\pi\left(X^{N-1} \times_{i}\{\bar{x}\}\right)=\pi\left(X_{*}^{N-1} \times_{i}\{\bar{x}\}\right),
$$

where the notation $E^{N-1} \times{ }_{i} F$ means $E^{i-1} \times F \times E^{N-i}$. Notice that the $N$ sets $X_{*}^{N-1} \times_{i}\{\bar{x}\}$ are disjoint; therefore, adding over $i=1, \ldots, N$, we get

$$
N \rho(\{\bar{x}\})=\sum_{i=1}^{N} \pi\left(X_{*}^{N-1} \times_{i}\{\bar{x}\}\right)=\pi\left(\bigcup_{i=1}^{N} X_{*}^{N-1} \times_{i}\{\bar{x}\}\right) \leq \pi\left(X^{N}\right)=1,
$$

from which $a(\rho) \leq 1 / N$.

Proposition 3.3. Let $\mathscr{C}$ be the cost introduced in (1.4) under the assumptions (1.1), (1.2), and (1.3), and let $a(\rho)$ be as in (1.6). Let $\rho \in \mathscr{P}(X)$ and $\bar{x} \in X$ such that $\rho(\{\bar{x}\})=\frac{1}{N}$. Then, letting $X_{*}=X \backslash\{\bar{x}\}$ and $\tilde{\rho}=\left.\frac{N}{N-1} \rho\right|_{X_{*}}$, we have

$$
\mathscr{C}_{N}(\rho)=(N-1) \int_{X_{*}} c(\bar{x}, y) \mathrm{d} \rho(y)+\mathscr{C}_{N-1}(\tilde{\rho}) .
$$

Proof. Let $\pi \in \Pi_{N-1}(\tilde{\rho})$; then we have that $P_{\text {sym }}\left(\delta_{\bar{x}} \otimes \pi\right) \in \Pi_{N}(\rho)$, and so

$$
\mathscr{C}_{N}(\rho) \leq C_{N}\left(P_{\text {sym }}\left(\delta_{\bar{x}} \otimes \pi\right)\right)=C_{N}\left(\delta_{\bar{x}} \otimes \pi\right)=(N-1) \int_{X_{*}} c(\bar{x}, y) \mathrm{d} \rho(y)+C_{N-1}(\pi) ;
$$

taking the infimum in $\pi$, we obtain the first inequality.

In order to prove the other inequality, we can assume $\mathscr{C}_{N}(\rho)<\infty$. Let us consider $\pi$ is a symmetric optimal coupling for $\mathscr{C}_{N}(\rho)$, which is therefore concentrated outside the diagonals, and let us define $X_{i}=X_{*}^{N-1} \times_{i}\{\bar{x}\}$; we know that $X_{i}$ are disjoint and $\pi\left(X_{i}\right)=P_{\#}^{i} \pi(\{\bar{x}\})=\frac{1}{N}$. This means that $\pi$ is concentrated on $\bigcup X_{i}$.

We can define $\pi_{1}$ through the implicit equality

$$
\left.\pi\right|_{\{\bar{x}\} \times X^{N-1}}=\left.\pi\right|_{X_{1}}=\frac{1}{N} \delta_{\bar{x}} \times \pi_{1}
$$

with $\pi_{1}\left(X_{*}^{N-1}\right)=\pi_{1}\left(X^{N-1}\right)=1$, and, thanks to the symmetry of $\pi$, by considering only permutations which fix the first coordinate, we deduce that also $\pi_{1}$ is symmetric in its $N-1$ variables. A simple computation then shows that

$$
\pi=P_{\text {sym }}\left(\delta_{\bar{x}} \otimes \pi_{1}\right)
$$

and that

$$
P_{\#}^{i}\left(\pi_{1}\right)=\tilde{\rho}=\left.\frac{N}{N-1} \rho\right|_{X_{*}} \quad \text { for every } i=1, \ldots, N-1 .
$$

Indeed, for every permutation which fixes $x_{1}$, we know that the measure is unchanged. On the other side, every permutation of coordinates can be written as the composition of one of these permutations with a $p^{i}: X^{N} \rightarrow X^{N}$ which exchanges $x_{1}$ and $x_{i}$ for some $i=2, \ldots, N$ and leaves all other coordinates fixed. For every $i=2, \ldots, N$, we know that $p_{\#}^{i}\left(\delta_{\bar{x}} \otimes \pi_{1}\right)$ goes to a nonnegative measure of total mass $1 / N$, concentrated on $X_{i}$ and hence orthogonal to $\delta_{\bar{x}} \otimes \pi_{1}$ (and to any other permutation $p^{j}$ with $j \neq i$ ), which is also a submeasure of $\pi$. Hence, we conclude that (3.2) holds. Define $\tilde{\rho}$ to 
be any marginal of $\pi_{1}$, so that $\delta_{\bar{x}} \otimes \pi_{1}$ has all marginals equal to $\tilde{\rho}$ apart from one, which equals $\delta_{\bar{x}}$. Symmetrizing, we find that $\rho$, which is any marginal of $\pi$, equals $1 / N \delta_{\bar{x}}+(N-1) / N \tilde{\rho}$, which proves $(3.3)$.

Then, thanks to (3.2) and (3.3), we can rewrite the energy of $\pi$ as

$$
\begin{aligned}
\mathscr{C}_{N}(\rho) & =C_{N}\left(\delta_{\bar{x}} \otimes \pi_{1}\right)=(N-1) \int_{X_{*}} c(\bar{x}, y) \mathrm{d} \rho(y)+C_{N-1}\left(\pi_{1}\right) \\
& \geq(N-1) \int_{X_{*}} c(\bar{x}, y) \mathrm{d} \rho(y)+\mathscr{C}_{N-1}(\tilde{\rho}) .
\end{aligned}
$$

At the threshold level $1 / N$ anything can happen: the cost can be finite or infinite, depending on the specific distribution of the mass.

Remark 3.4. If $X$ is a space with at least one accumulation point, and $\mathscr{C}$ and $a$ are as in Proposition 3.3, then there exists $\rho \in \mathscr{P}(X)$ such that $a(\rho)=1 / N$ and $\operatorname{supp}(\pi) \cap D \neq \emptyset$ for every $\pi \in \Pi(\rho)$ (thus $\pi\left(D_{\alpha}\right)>0$ for every $\alpha>0$ ). This shows that the assumption on the concentration in Theorem 1.3 is necessary. Moreover, there is one such $\rho$ with $\mathscr{C}(\rho)<\infty$ and one with $\mathscr{C}(\rho)=\infty$.

Indeed, let $x \in X$ be a limit point, $\left(x_{n}\right)_{n \in \mathbb{N}} \subset X \backslash\{x\}$ be a sequence of distinct points converging to $x$, and

$$
\rho:=\frac{1}{N} \delta_{x}+\frac{N-1}{N} \sum_{n} p_{n} \delta_{x_{n}}
$$

where $\left(p_{n}\right)_{n \in \mathbb{N}} \in \ell^{1}$ with $p_{n} \in(0,1 / N)$ and $\sum_{n=1}^{\infty} p_{n}=1$.

With the notation of the proof of Proposition 3.3, we have

$$
\begin{aligned}
\pi\left(D_{\alpha}\right) & \geq \pi\left(\{x\} \times X_{*}^{N-2} \times B(x, \alpha)\right)=\pi_{1}\left(X_{*}^{N-2} \times B(x, \alpha)\right) \\
& =P_{\#}^{n-1} \pi_{1}(B(x, \alpha))=\tilde{\rho}(B(x, \alpha))>0 .
\end{aligned}
$$

Finally, since $a(\tilde{\rho})<\frac{1}{N}<\frac{1}{N-1}$, we have $\mathscr{C}_{N-1}\left(\sum_{n} p_{n} \delta_{x_{n}}\right)<\infty$, and so, again by Proposition $3.3, \mathscr{C}_{N}(\rho)$ is finite if and only if

$$
\int_{X_{*}} c(\bar{x}, y) \mathrm{d} \rho(y)=\sum_{n} p_{n} c\left(\bar{x}, x_{n}\right)
$$

is finite; one can choose the weights $\left(p_{n}\right)_{n}$ appropriately, taking into account (1.2), in order to make the cost finite or infinite.

Proof of Theorem 1.1(iii). We argue inductively on the number $i$ of atoms in $\mu$ of mass $1 / N$. If $\mu$ has exactly one atom of mass $1 / N$ at $\bar{x}$, namely, if $i=1$, from Proposition 3.3 we know that the cost of $\mathscr{C}_{N}$ is finite if and only if condition (1.7) is in force at $\bar{x}$ and $\left.\frac{N}{N-1} \rho\right|_{X \backslash\{\bar{x}\}}$ has finite $\mathscr{C}_{N-1}$ cost. On the other side, this second condition is always verified because $\left.\frac{N}{N-1} \rho\right|_{X \backslash\{\bar{x}\}}$ does not have atoms of the critical mass $1 /(N-1)$; hence the first part of Theorem 1.1 applies.

If we assume the statement to be true when there are $i$ atoms in $\mu$ of mass $1 / N$ and we want to prove it for $i+1$, we consider $\mu$ with $i+1$ atoms of mass $1 / N$, one of which at $\bar{x}$; next we apply Proposition 3.3 , and we reduce to studying the finiteness of the cost of $\left.\frac{N}{N-1} \rho\right|_{X \backslash\{\bar{x}\}}$, which in turn has exactly $i$ atoms of mass $1 /(N-1)$. Hence, applying the inductive assumption to this measure, we conclude the proof. 
For simplicity we give the following example of discontinuity of the cost for the Coulomb cost in $\mathbb{R}^{d}$; of course, it can be generalized to metric spaces with costs as in assumptions (1.1) and (1.2).

Proposition 3.5. Let $c(x, y)=|x-y|^{-1}$ in $\mathbb{R}^{d} \times \mathbb{R}^{d}$, and consider the minimization problem (1.4). Let $\rho \in \mathscr{P}\left(\mathbb{R}^{d}\right)$ be such that $a(\rho)=\frac{1}{N}$. Then for every $\varepsilon>0$ small there exists $\tilde{\rho}$ such that $|\rho-\tilde{\rho}|\left(\mathbb{R}^{d}\right) \leq \varepsilon$ and

$$
1+\mathscr{C}(\rho)<\mathscr{C}(\tilde{\rho})<\infty .
$$

Proof. Let $\bar{x} \in X$ and $\mu \in \mathscr{M}_{+}(X)$ be such that $\rho=\frac{1}{N} \delta_{\bar{x}}+\mu$. Then by Proposition 3.3 we know that

$$
N \int_{\mathbb{R}^{d}} c(\bar{x}, y) \mathrm{d} \mu(y)+\mathscr{C}_{N-1}\left(\frac{N}{N-1} \mu\right)=\mathscr{C}_{N}(\rho)<\infty .
$$

Let $\varepsilon<1 / N$ and $y_{\varepsilon} \in \mathbb{R}^{d}$ such that $\varepsilon<\left|\bar{x}-y_{\varepsilon}\right| \leq 2 \varepsilon$ and $\mu\left(y_{\varepsilon}\right)=0$. We consider

$$
\rho_{\varepsilon}:=\frac{1}{N} \delta_{\bar{x}}+\varepsilon \delta_{y_{\varepsilon}}+(1-\varepsilon) \mu \in \mathscr{P}\left(\mathbb{R}^{d}\right),
$$

we notice that $\left|\rho-\rho_{\varepsilon}\right|\left(\mathbb{R}^{d}\right)=\varepsilon\left|-\delta_{y_{\varepsilon}}+\varepsilon \mu\right|\left(\mathbb{R}^{d}\right) \leq 2 \varepsilon$, and we estimate its cost thanks to Proposition 3.3 as

$$
\mathscr{C}_{N}\left(\rho_{\varepsilon}\right)=N \int_{\mathbb{R}^{d}} c(\bar{x}, y) \mathrm{d}\left(\varepsilon \delta_{y_{\varepsilon}}+(1-\varepsilon) \mu\right)(y)+\mathscr{C}_{N-1}\left(\frac{N}{N-1}\left(\varepsilon \delta_{y_{\varepsilon}}+(1-\varepsilon) \mu\right)\right) .
$$

The last term is finite because $a\left(\varepsilon \delta_{y_{\varepsilon}}+(1-\varepsilon) \mu\right) \leq \frac{1-\varepsilon}{N}$, and the first one thanks to (3.5). On the other side, we estimate the cost from below by

$$
\begin{aligned}
\mathscr{C}_{N}\left(\rho_{\varepsilon}\right) & \geq N \varepsilon c\left(\bar{x}, y_{\varepsilon}\right)+N(1-\varepsilon) \int_{\mathbb{R}^{d}} c(\bar{x}, y) \mathrm{d} \mu(y)+\mathscr{C}_{N-1}\left(\frac{N}{N-1}\left(\varepsilon \delta_{y_{\varepsilon}}+(1-\varepsilon) \mu\right)\right) \\
& \geq \frac{N}{2}+N(1-\varepsilon) \int_{\mathbb{R}^{d}} c(\bar{x}, y) \mathrm{d} \mu(y)+\mathscr{C}_{N-1}\left(\frac{N}{N-1}\left(\varepsilon \delta_{y_{\varepsilon}}+(1-\varepsilon) \mu\right)\right) .
\end{aligned}
$$

By the lower semicontinuity of the cost $\mathscr{C}_{N-1}$, we know that the last two terms on the right-hand side are, in the limit, greater than or equal to the quantity in (3.5), namely $\mathscr{C}_{N}(\rho)$. Hence, for $\varepsilon$ small enough we obtain (3.4).

\section{Uniform bounds on the cost and diagonal bounds.}

TheOREM 4.1 (uniform bound on the cost in terms of the concentration). Let $\rho \in$ $\mathscr{P}(X)$ be such that $\kappa(\rho, r) \leq \frac{1}{N}$ for some $r>0$. Let $r(x)$ be such that $\rho(B(x, r(x))) \leq$ $1 / N$ for every $x$. Then we have

$$
\mathscr{C}(\rho) \leq\left(\begin{array}{c}
N \\
2
\end{array}\right) \int M\left(\max \left\{\frac{r(x)}{2}, r\right\}\right) \mathrm{d} \rho .
$$

Remark 4.2. Under the assumptions of Theorem 4.1, the slightly weaker bound

$$
\mathscr{C}(\rho) \leq\left(\begin{array}{c}
N \\
2
\end{array}\right) M(r)
$$

can be achieved in a simpler way. Indeed, this is a straightforward application of Theorem 4.3 with the set $D=\{d(x, y)<r\}$, which guarantees us the existence of a plan $\pi \in \Pi_{N}(\rho)$, concentrated outside $D_{r}$; in particular we have $c\left(x_{i}, x_{j}\right) \leq M(r)$ for every $i \neq j$, for $\pi$-a.e., and so we have $C(\pi) \leq\left(\begin{array}{c}N \\ 2\end{array}\right) M(r)$. 
Proof of Theorem 4.1. Without loss of generality we can assume that $r(x)$ is the maximum radius such that $\rho(B(x, r(x))) \leq 1 / N$. We first notice that $r(x)$ is 1 Lipschitz; in fact, since $B(x, r(x)+\varepsilon) \subseteq B(y, r(x)+\varepsilon+d(x, y))$ by the maximality of $r(x)$ we deduce that $\rho(B(y, r(x)+\varepsilon+d(x, y)))>\frac{1}{N}$. This is true for every $\varepsilon>0$, so we get

$$
r(y) \leq r(x)+d(x, y)
$$

Since we can reverse the roles of $x$ and $y$, we obtain that $r$ is 1-Lipschitz.

Let $D=\{(x, y): 2 d(x, y)<\max \{r(x), r(y), 2 r\}\}$; since both $d$ and $r$ are continuous, we have that $D$ is an open symmetric set. Moreover, defining $B(x)=\left\{x^{\prime}\right.$ : $\left.\left(x, x^{\prime}\right) \in D\right\}$ we have

$$
\begin{aligned}
B(x) & =B(x, r) \cup B\left(x, \frac{r(x)}{2}\right) \cup\left\{x^{\prime}: d\left(x, x^{\prime}\right)<\frac{r\left(x^{\prime}\right)}{2}\right\} \\
& \subseteq B(x, r) \cup B\left(x, \frac{r(x)}{2}\right) \cup\left\{x^{\prime}: d\left(x, x^{\prime}\right)<\frac{d\left(x, x^{\prime}\right)+r(x)}{2}\right\}=B(x, r) \cup B(x, r(x)),
\end{aligned}
$$

where we used the fact that $r$ is 1-Lipschitz. Clearly we thus have $\rho(B(x)) \leq \frac{1}{N}$; so we can use Theorem 4.3 in order to get a plan $\pi \in \Pi_{N}(\rho)$ such that $\left(x_{i}, x_{j}\right) \notin D$ for $i \neq j$, for $\pi$-a.e., $x \in X^{k}$. But this means that $d\left(x_{i}, x_{j}\right) \geq \max \left\{r\left(x_{i}\right), r\left(x_{j}\right)\right\} / 2$ for $\pi$-a.e. $x$ : we then get

$$
\begin{aligned}
\mathscr{C}(\rho) \leq C(\pi) & \leq \int_{X^{N}} \sum_{i<j} M\left(\frac{\max \left\{r\left(x_{i}\right), r\left(x_{j}\right)\right\}, 2 r}{2}\right) \mathrm{d} \pi \\
& \leq \int_{X} \sum_{i<j} \frac{M\left(\max \left\{\frac{r\left(x_{i}\right)}{2}, r\right\}\right)+M\left(\max \left\{\frac{r\left(x_{j}\right)}{2}, r\right\}\right)}{2} \mathrm{~d} \pi \\
& =\left(\begin{array}{c}
N \\
2
\end{array}\right) \int_{X} M\left(\max \left\{\frac{r(x)}{2}, r\right\}\right) \mathrm{d} \rho .
\end{aligned}
$$

In graph theory, a consequence of the Hajnal-Szemerédi theorem [21, 23] is a simplified multimarginal version of the marriage theorem (a multimarriage theorem): let us suppose we have $k N$ people, and everyone has a list of hated people, which always has fewer than $k$ people on it (hatred is a reciprocal sentiment, at least in this example). Then we can form $k$ groups of $N$ people such that in every group we do not have people who hate each other.

The following theorem can be seen as the continuous analogue of this multimarriage theorem $(B(x)$ is the list of people disliked by $x)$.

Theorem 4.3 (existence of a plan outside the diagonal). Let $X$ be a Polish space, and let $D \subset X^{2}$ be a symmetric open set; let us denote $B(x)=\left\{x^{\prime}:\left(x, x^{\prime}\right) \in D\right\}$. Let us suppose that $\rho(B(x)) \leq 1 / N$ for every $x \in X$; then there exists a plan $\pi \in \Pi_{N}(\rho)$ that is concentrated on $A^{c}$, where

$$
A=\bigcup_{X^{N}} \bigcup_{i \neq j}\left\{\left(x_{i}, x_{j}\right) \in D\right\}
$$

that is, we have $\left(x_{i}, x_{j}\right) \notin D$ for $i \neq j$, for $\pi$-a.e. $x \in X^{N}$.

Proof. The proof exploits the duality formula for bounded costs. In order to show that there exists an admissible plan $\pi \in \Pi_{N}(\rho)$ such that $\pi(A)=0$, we will analyze 
the minimizer of the multimarginal optimal transport with respect to the following bounded cost:

$$
\tilde{c}\left(x_{1}, \ldots, x_{N}\right)=\inf \left\{d\left(\left(x_{1}, \ldots, x_{N}\right), A^{c}\right), 1\right\} .
$$

For this cost it is known that the duality formula holds [13]:

$$
\inf _{\pi \in \Pi(\rho)} \int_{X^{N}} \tilde{c} \mathrm{~d} \pi=\sup _{\varphi\left(x_{1}\right)+\cdots+\varphi\left(x_{N}\right) \leq \tilde{c}(x)} N \int_{X} \varphi \mathrm{d} \rho .
$$

The optimal $\pi \in \Pi_{N}(\rho)$ will satisfy $\pi(A)=0$ if we show that

$$
\int_{X} \varphi \mathrm{d} \rho \leq 0 \quad \text { for all admissible } \varphi .
$$

In fact, in such a case the optimal value of the previous problems must be 0 ; therefore, $\pi$ has to be supported on $A^{c}$, thanks to the fact that $A$ is open.

Actually, the crucial constraint on $\varphi$ that will be needed for the proof is

$$
\varphi\left(x_{1}\right)+\cdots+\varphi\left(x_{N}\right) \leq 0 \quad \text { if } x \in A^{c} .
$$

The only role that the cost $\tilde{c}$ on $A$ plays is telling us that $\varphi$ is bounded from above, since $N \varphi(x) \leq \tilde{c}(x, \ldots, x) \leq 1$; indeed, one would like to consider the cost which takes the value $\infty$ in this region if there were not the problem of the validity of the duality formula for such a cost and the boundedness of the potential.

After having fixed a small $\varepsilon>0$, we do the following iterative construction of $\eta_{i}, z_{i}$, and $B_{i}$. For $i=1$, we define $\eta_{1}=\sup _{X} \varphi$, we select $z_{1} \in X$ such that $\varphi\left(z_{1}\right) \geq \eta_{1}-\varepsilon$, and we let $B_{1}=B\left(z_{1}\right)$; for $i>1$ we proceed analogously according to the following:

$$
\begin{array}{ccc}
\eta_{1}=\sup _{X} \varphi, & z_{1} \in X, \varphi\left(z_{1}\right) \geq \eta_{1}-\varepsilon, & B_{1}=B\left(z_{1}\right), \\
\eta_{2}=\sup _{B_{1}^{c}} \varphi, & z_{2} \in B_{1}^{c}, \varphi\left(z_{2}\right) \geq \eta_{2}-\varepsilon, & B_{2}=B\left(z_{2}\right), \\
\vdots & \vdots & \vdots \\
\eta_{k}=\sup _{\left(B_{1} \cup \cdots \cup B_{k-1}\right)^{c}} \varphi, & z_{k} \in\left(B_{1} \cup \cdots \cup B_{k-1}\right)^{c}, \varphi\left(z_{k}\right) \geq \eta_{k}-\varepsilon, & B_{k}=B\left(z_{k}\right) .
\end{array}
$$

Notice that the sequence $r / N \geq \eta_{1} \geq \eta_{2} \geq \ldots$ is monotone.

At each step we check the sign of the quantity

$$
\eta_{1}+\cdots+\eta_{k-1}+(N-k+1) \eta_{k}-(k-1) \varepsilon .
$$

As soon as it is nonpositive we stop the process and estimate the quantity $\int_{X} \varphi \mathrm{d} \rho$. Notice that this will surely happen by the time we reach $k=N$, because if $z \in$ $\left(B_{1} \cup \cdots \cup B_{N-1}\right)^{c}$, then $\left(z_{1}, \ldots, z_{N-1}, z\right) \in A^{c}$ (in fact, letting $z_{N}:=z$, we have $\left(z_{i}, z_{j}\right) \notin D$ for every $\left.i \neq j\right)$, so

$$
\left(\eta_{1}-\varepsilon\right)+\cdots+\left(\eta_{N-1}-\varepsilon\right)+\varphi(z) \leq \varphi\left(z_{1}\right)+\cdots+\varphi\left(z_{N-1}\right)+\varphi(z) \leq 0,
$$

and $\eta_{1}+\cdots+\eta_{N}-(N-1) \varepsilon \leq 0$ follows by taking the supremum over $z$.

Calling $k$ the smallest integer for which this happens, by construction we have

$$
\eta_{k} \leq-\frac{1}{N-k+1} \sum_{j=1}^{k-1} \eta_{j}+\frac{k-1}{N-k+1} \varepsilon,
$$

Copyright (C) by SIAM. Unauthorized reproduction of this article is prohibited. 
while the preceding inequalities are reversed.

Letting $\tilde{B}_{j}=B_{j} \backslash\left(B_{1} \cup \cdots \cup B_{k-1}\right)$ so that they are disjoint, we can estimate

$$
\begin{aligned}
\int_{X} \varphi \mathrm{d} \rho & =\sum_{i=1}^{k-1} \int_{\tilde{B}_{i}} \varphi \mathrm{d} \rho+\int_{\left(B_{1} \cup \cdots \cup B_{k-1}\right)^{c}} \varphi \mathrm{d} \rho \\
& \leq \sum_{i=1}^{k-1} \eta_{i} \rho\left(\tilde{B}_{i}\right)+\eta_{k}\left(1-\sum_{i=1}^{k-1} \rho\left(\tilde{B}_{i}\right)\right) \\
& \leq \sum_{i=1}^{k-1} \eta_{i} \rho\left(\tilde{B}_{i}\right)+\left(-\frac{1}{N-k+1} \sum_{j=1}^{k-1} \eta_{j}+\frac{k-1}{N-k+1} \varepsilon\right)\left(1-\sum_{i=1}^{k-1} \rho\left(\tilde{B}_{i}\right)\right) \\
& =\sum_{i=1}^{k-1} \rho\left(\tilde{B}_{i}\right) \underbrace{\left(\eta_{i}+\frac{1}{N-k+1} \sum_{j=1}^{k-1} \eta_{j}-\frac{k-2}{N-k+1} \varepsilon\right)}_{\geq 0} \\
& -\frac{1}{N-k+1} \sum_{j=1}^{k-1} \eta_{j}+\frac{k-1}{N-k+1} \varepsilon-\frac{1}{N-k+1} \varepsilon \sum_{i=1}^{k-1} \rho\left(\tilde{B}_{i}\right),
\end{aligned}
$$

where we used (4.1) in the second inequality and then

$$
\begin{aligned}
& (N-k+1) \eta_{i}+\sum_{j=1}^{k-1} \eta_{j}-(k-2) \varepsilon \\
& \geq(N-k+1) \eta_{k-1}+\sum_{j=1}^{k-1} \eta_{j}-(k-2) \varepsilon \\
& \quad=(N-(k-1)+1) \eta_{k-1}+\sum_{j=1}^{k-2} \eta_{j}-(k-2) \varepsilon \geq 0
\end{aligned}
$$

in the underlined part in the last equation; in particular, we can use $\rho\left(B_{i}\right) \leq 1 / N$ for $i<k$ :

$$
\begin{aligned}
\int_{X} \varphi d \rho & \leq \sum_{i=1}^{k-1} \frac{1}{N}\left(\eta_{i}+\frac{1}{N-k+1} \sum_{j=1}^{k-1} \eta_{j}-\frac{k-2}{N-k+1} \varepsilon\right)-\frac{1}{N-k+1} \sum_{j=1}^{k-1} \eta_{j}+\frac{k-1}{N-k+1} \varepsilon \\
& \leq\left(\frac{1}{N}+\frac{k-1}{N(N-k+1)}-\frac{1}{N-k+1}\right) \sum_{j=1}^{k-1} \eta_{j}+\frac{k-1}{N-k+1} \varepsilon \\
& =\frac{k-1}{N-k+1} \varepsilon \leq N \varepsilon .
\end{aligned}
$$

Letting $\varepsilon \rightarrow 0$ shows that $\int_{X} \varphi \mathrm{d} \rho \leq 0$ as desired.

Proof of Theorem 1.3(ii). First, we can assume without loss of generality that the plan $\pi$ is symmetric, since $\pi^{\mathrm{sym}}$ has the same cost of $\pi$ and $\pi^{\mathrm{sym}}\left(\bar{D}_{\alpha}\right)=\pi\left(\bar{D}_{\alpha}\right)$ for every $\alpha \geq 0$.

Assume by contradiction that $\pi\left(\bar{D}_{\alpha}\right)>0$. Then there exists $x \in \operatorname{supp}(\pi) \cap D_{\alpha}$. We may assume without loss of generality that $\left|x_{1}-x_{2}\right| \leq \alpha$. For notational simplicity, let $\gamma=\beta / 2 \leq r$. We claim that there is a point

$$
y \in \operatorname{supp}(\pi) \backslash \bar{D}_{\beta} \cap\left(\bar{B}\left(x_{1}, \gamma\right)^{c}\right)^{N} .
$$

Copyright (c) by SIAM. Unauthorized reproduction of this article is prohibited. 
To prove that such a point exists, it is sufficient to show that

$$
\pi\left(\bar{D}_{\beta}^{c} \cap\left(\bar{B}\left(x_{1}, \gamma\right)^{c}\right)^{N}\right)>0 .
$$

But this is true since we can estimate the mass of the complement as

$$
\begin{aligned}
\pi\left(\left[\bar{D}_{\beta}^{c} \cap\left(\bar{B}\left(x_{1}, \gamma\right)^{c}\right)^{N}\right]^{c}\right) & =\pi\left(\bar{D}_{\beta} \cup\left[\left(\bar{B}\left(x_{1}, \gamma\right)^{c}\right)^{N}\right]^{c}\right) \\
& \leq \pi\left(\bar{D}_{\beta}\right)+\pi\left(\bigcup_{i=1}^{N} X^{N-1} \times_{i} \bar{B}\left(x_{1}, \gamma\right)\right) \\
& \leq \frac{C(\pi)}{m(\beta)}+N \rho\left(\bar{B}\left(x_{1}, \gamma\right)\right) \\
& <1-N \kappa(\rho, r)+N \kappa(\rho, \gamma) \leq 1 .
\end{aligned}
$$

Next we prove that there exists $i \in\{1, \ldots, N\}$ such that $d\left(y_{i}, x_{j}\right)>\gamma$ for every $j=1, \ldots, N$. Indeed, by definition of $y$, the set $\bar{B}\left(x_{1}, \gamma\right)$ does not contain any of the points $y_{i}$; furthermore, the $N-1$ sets $\bar{B}\left(x_{2}, \gamma\right), \bar{B}\left(x_{3}, \gamma\right), \ldots, \bar{B}\left(x_{N}, \gamma\right)$ have diameter at most $2 \gamma=\beta$; therefore, at least one of the $N$ points $y_{i}$ does not belong to any of them; otherwise, by the pigeonhole principle one of the aforementioned sets would contain two of the points $y_{i}$, which is impossible because they are pairwise spaced apart by more than $\beta$. Since we are dealing with a symmetric plan, we may assume that $d\left(y_{1}, x_{j}\right)>\gamma$ for every $j=1, \ldots, N$.

Now we introduce the two points $\tilde{x}$ and $\tilde{y}$ obtained by swapping the coordinates $x_{1}$ and $y_{1}$, namely

$$
\tilde{x}=\left(y_{1}, x_{2}, \ldots, x_{N}\right), \quad \tilde{y}=\left(x_{1}, y_{2}, \ldots, y_{N}\right) .
$$

Thanks to the $c$-monotonicity we then have $c(x)+c(y) \leq c(\tilde{x})+c(\tilde{y})$. In this last inequality many terms cancel out; in fact, the interaction between $x_{i}$ and $x_{j}$ for $i, j \geq 2$ and between $y_{i}$ and $y_{j}$ for $i, j \geq 2$ are present on both sides. Thus, the inequality is equivalent to

$$
\sum_{i=2}^{N} c\left(x_{1}, x_{i}\right)+c\left(y_{1}, y_{i}\right) \leq \sum_{i=2}^{N} c\left(y_{1}, x_{i}\right)+c\left(x_{1}, y_{i}\right) .
$$

Now we can use $d\left(x_{1}, x_{2}\right) \leq \alpha, d\left(x_{1}, y_{i}\right)>\gamma$, and $d\left(y_{1}, x_{i}\right)>\gamma$ to get

$$
m(\alpha) \leq \sum_{i=2}^{N} c\left(x_{1}, x_{i}\right)+c\left(y_{1}, y_{i}\right) \leq \sum_{i=2}^{N} c\left(y_{1}, x_{i}\right)+c\left(x_{1}, y_{i}\right) \leq 2(N-1) M(\gamma),
$$

and so we have reached a contradiction.

Proof of Theorem 1.3(i). As in the proof of Theorem 1.3(ii) we can assume that $\pi$ is symmetric. Assume by contradiction that $\pi\left(\bar{D}_{\alpha}\right)>0$. Then there exists $x \in$ $\operatorname{supp}(\pi) \cap D_{\alpha}$. We may assume without loss of generality that $d\left(x_{1}, x_{2}\right) \leq \alpha$. We claim that there is a point $y \in \operatorname{supp}(\pi)$ such that

$$
y \in\left(\bigcup_{i=2}^{N} \bar{B}\left(x_{i}, r\right)\right)^{c} \times\left(\bar{B}\left(x_{1}, r\right)^{c}\right)^{N-1} .
$$

Copyright $@$ by SIAM. Unauthorized reproduction of this article is prohibited. 
For notational convenience, let us denote $A_{1}=\bigcup_{i=2}^{N} \bar{B}\left(x_{i}, r\right)$ and $A=\bar{B}\left(x_{1}, r\right)$. To prove that such a point exists, it is sufficient to show that

$$
\pi\left(A_{1}^{c} \times\left(A^{c}\right)^{N-1}\right)>0 .
$$

But this is true since we can estimate the mass of the complement as

$$
\begin{aligned}
\pi\left(\left[A_{1}^{c} \times\left(A^{c}\right)^{N-1}\right]^{c}\right) & \leq \pi\left(A_{1} \times X^{N-1} \cup \bigcup_{i=2}^{N} A \times_{i} X^{N-1}\right) \\
& \leq \rho\left(A_{1}\right)+(N-1) \rho(A) \\
& \leq \sum_{i=2}^{N} \rho\left(B\left(x_{i}, r\right)\right)+(N-1) \rho\left(B\left(x_{1}, r\right)\right)<1 .
\end{aligned}
$$

Now we introduce the two points $\tilde{x}$ and $\tilde{y}$ obtained by swapping the coordinates $x_{1}$ and $y_{1}$, namely

$$
\tilde{x}=\left(y_{1}, x_{2}, \ldots, x_{N}\right), \quad \tilde{y}=\left(x_{1}, y_{2}, \ldots, y_{N}\right) .
$$

Thanks to the $c$-monotonicity we then have $c(x)+c(y) \leq c(\tilde{x})+c(\tilde{y})$. In this last inequality many terms cancel out; in fact, the interactions between $x_{i}$ and $x_{j}$ for $i, j \geq 2$ and between $y_{i}$ and $y_{j}$ for $i, j \geq 2$ are present on both sides. Thus, the inequality is equivalent to

$$
\sum_{i=2}^{N} c\left(x_{1}, x_{i}\right)+c\left(y_{1}, y_{i}\right) \leq \sum_{i=2}^{N} c\left(y_{1}, x_{i}\right)+c\left(x_{1}, y_{i}\right)
$$

Now we can use $d\left(x_{1}, x_{2}\right) \leq \alpha, d\left(x_{1}, y_{i}\right)>r$ and $d\left(y_{1}, x_{i}\right)>r$ to get

$$
m(\alpha) \leq \sum_{i=2}^{N} c\left(x_{1}, x_{i}\right)+c\left(y_{1}, y_{i}\right) \leq \sum_{i=2}^{N} c\left(y_{1}, x_{i}\right)+c\left(x_{1}, y_{i}\right) \leq 2(N-1) M(r),
$$

and so we have reached a contradiction.

5. Estimates on the potentials and continuity of the cost. Putting together the previous results, it is possible to show the continuity of the cost function $\mathscr{C}$ under a more general hypothesis than the one assumed in [5], following the same strategy; we sketch the short argument for completeness. Moreover, as Remark 3.4 tells us, the threshold $1 / N$ is sharp. An important role will be played by truncated cost, which we therefore introduce: we define

$$
c^{h}(x, y):=\min \{c(x, y), h\} .
$$

Then, similarly to (1.3)-(1.4) we define $c^{h}\left(x_{1}, \ldots, x_{N}\right)=\sum_{i<j} c^{h}\left(x_{i}, x_{j}\right)$ and

$$
C^{h}(\pi)=\int_{X^{N}} c^{h} \mathrm{~d} \pi, \quad \mathscr{C}^{h}(\rho)=\min \left\{C^{h}(\pi): \pi \in \Pi(\rho)\right\} .
$$

The following lemma uses the diagonal bounds on the truncated cost to prove that for $h$ sufficiently big we have that the minimizing problems with $\mathscr{C}^{h}$ and $\mathscr{C}$ have in fact the same minimizers. 
LEMMA 5.1 (equivalence with a truncated cost). Let us consider a cost c satisfying assumptions (1.1) and (1.2), and let $r>0$ and $\delta<\frac{1}{N}$. Then there exists $h=h(r, \delta)$ such that for every $\rho$ belonging to the set $\mathscr{K}_{r, \delta}$ introduced in (1.8) we have the following:

(i) $\pi$ is a minimizer for $\mathscr{C}^{h}(\rho)$ if and only if it is a minimizer for $\mathscr{C}(\rho)$.

(ii) If $\varphi^{h}$ is an optimal potential for $\mathscr{C}^{h}(\rho)$, then it is also an optimal potential for $\mathscr{C}(\rho)$.

In particular, we have that $\mathscr{C}=\mathscr{C}^{h}$ on $\mathscr{K}_{r, \delta}$.

Proof. Let us consider $\rho \in \mathscr{K}_{r, \delta}$.

(i) Let us consider a plan $\pi$ which is optimal for the problem $\mathscr{C}(\rho)$ and a plan $\pi^{h}$ which is optimal for $\mathscr{C}^{h}(\rho)$. First we prove that we can choose $\beta$ in Theorem 1.3(ii) depending only on $r, \delta$ and not on $\rho$ specifically. In fact, we could consider $\beta$ such that

$$
m(\beta)>\frac{\left(\begin{array}{c}
N \\
2
\end{array}\right) M(r)}{1-N \delta}
$$

and this is sufficient to satisfy (1.9); in fact, thanks to Remark 4.2, we have $\mathscr{C}(\rho) \leq\left(\begin{array}{c}N \\ 2\end{array}\right) M(r)$, and so in particular

$$
m(\beta)>\frac{\left(\begin{array}{c}
N \\
2
\end{array}\right) M(r)}{1-N \delta} \geq \frac{\mathscr{C}(\rho)}{1-N \kappa(\rho, r)} .
$$

Now, fix $h>h^{\prime}>\max \{2(N-1) M(\beta / 2), m(\beta)\}$ and define $m^{h}(r)=\min \{m(r), h\}$ and $M^{h}(r)=\min \{M(r), h\}$. We apply again Theorem 1.3(ii) with the cost $c^{h}$, $m^{h}(r)$, and $M(r)$, which satisfy assumption (1.1) too. Notice that $h>m(\beta)$ by construction, and in particular we have $m(\beta)=m^{h}(\beta)$, and so we get

$$
m^{h}(\beta)=m(\beta)>\frac{\left(\begin{array}{c}
N \\
2
\end{array}\right) M(r)}{1-N \delta} \geq \frac{\mathscr{C}^{h}(\rho)}{1-N \kappa(\rho, r)} .
$$

Since $h^{\prime}>2(N-1) M(\beta / 2)$, we must have $\pi_{h}\left(D_{c^{h}}^{h^{\prime}}\right)=0$ for every $\pi_{h}$ optimal plan for the problem $\mathscr{C}^{h}(\rho)$. However, since $h^{\prime}<h$, it is clear that whenever $c^{h}(x, y) \leq h^{\prime}$ we have $c^{h}(x, y)=c(x, y)$. But then we have $c=c^{h}$ on the support of $\pi_{h}$, and so

$$
\int c^{h} \mathrm{~d} \pi_{h}=\int c \mathrm{~d} \pi_{h} \geq \int c \mathrm{~d} \pi \geq \int c^{h} \pi \geq \int c^{h} \mathrm{~d} \pi_{h},
$$

where all the other inequalities are true for the optimality, or from $c \geq c^{h}$. Since the first term and the last term are equal, we deduce that they are all equal, and, in particular, $\pi$ is a minimizer also for $\mathscr{C}^{h}$ and $\pi_{h}$ is a minimizer also for $\mathscr{C}$, concluding the proof.

(ii) First, if $\varphi^{h}$ is an optimal potential for $\mathscr{C}^{h}(\rho)$, then of course it is admissible also for $c \geq c^{h}$. Moreover, by (i), we have $\mathscr{C}(\rho)=\mathscr{C}^{h}(\rho)=\int \varphi^{h} \mathrm{~d} \rho$, proving also the maximality of $\varphi^{h}$.

We state explicitly what we can choose for $h(r, \delta)$ :

$$
h(r, \delta)>2(N-1) \cdot M\left(\frac{1}{2} m^{-1}\left(\frac{\left(\begin{array}{c}
N \\
2
\end{array}\right) M(r)}{1-N \delta}\right)\right) .
$$

This expression is very complicated since it is also in terms of $m, M$; however, if, for example, we have $M(r) \leq \alpha m(r)$ for some $\alpha>0$, we can choose a more explicit form 
for $h(r, \delta)$ :

$$
h(r, \delta)>\frac{\alpha N(N-1)^{2}}{1-N \delta} \cdot M(r) .
$$

In the following theorem we want to recall typical regularity results that the potentials can inherit from the cost.

Theorem 5.2 (regularity of the potential). Assume that $c: X \times X \rightarrow[0, \infty)$ is a lower semicontinuous bounded cost. Then for every $\rho \in \mathscr{P}(X)$ there exists an optimal potential $\varphi$ such that the following hold:

- $\varphi$ is bounded and

$$
-\frac{(N-1)^{2}}{2}\|c\|_{\infty} \leq \varphi(x) \leq \frac{N-1}{2}\|c\|_{\infty} .
$$

- If, moreover, $c$ is Lipschitz, then $\varphi$ is Lipschitz and

$$
\|\varphi\|_{\text {Lip }} \leq(N-1)\|c\|_{\text {Lip }} .
$$

- If $X$ is geodesic and $c$ is $K$-concave, then $\varphi$ can be assumed to be $(N-1) K$ concave.

Proof. Let $\varphi_{1}, \ldots, \varphi_{N}$ be admissible potentials. We will now construct new potentials $\tilde{\varphi}_{1}, \ldots, \tilde{\varphi}_{N}$ which will be admissible and satisfy the regularity assumption, and moreover $\varphi_{i} \leq \tilde{\varphi}_{i}$.

First we have that $\sup \varphi_{i}=t_{i}<\infty$; otherwise they are not admissible potentials; moreover, we have, for the admissibility, that $t_{1}+\cdots+t_{N} \leq\left(\begin{array}{c}N \\ 2\end{array}\right)\|c\|_{\infty}$.

We can then modify the potential $\varphi_{1}$ taking

$$
\tilde{\varphi}_{1}(x)=\inf \left\{c\left(x, \ldots, x_{n}\right)-\sum_{i=2}^{N} \varphi_{i}(x)\right\} .
$$

Of course, by construction $\left(\tilde{\varphi}_{1}, \varphi_{2}, \ldots, \varphi_{N}\right)$ are admissible potentials, and moreover we have $\varphi_{1} \leq \tilde{\varphi}_{1}$. Denoting $\tilde{t}_{1}=\sup \tilde{\varphi}_{1}$, in particular we can now say that

$$
\begin{gathered}
\tilde{\varphi}_{1}(x) \geq-t_{2}-\cdots-t_{N}=\tilde{t}_{1}-\left(\tilde{t}_{1}+\cdots+t_{N}\right) \geq \tilde{t}_{1}-\left(\begin{array}{c}
N \\
2
\end{array}\right)\|c\|_{\infty}, \\
\inf \tilde{\varphi}_{1}-\sup \tilde{\varphi}_{1} \geq-\left(\begin{array}{c}
N \\
2
\end{array}\right)\|c\|_{\infty} .
\end{gathered}
$$

Notice also that if $c$ is $L$-Lipschitz (respectively, $K$-concave), then $\tilde{\varphi}_{1}$ is an infimum of $(N-1) L$-Lipschitz (respectively, $(N-1) K$-concave) functions, and so we have that $\tilde{\varphi}_{1}$ is $(N-1) L$-Lipschitz (respectively, $(N-1) K$-concave).

We can iterate this construction in order to get $\tilde{\varphi}_{1}, \ldots, \tilde{\varphi}_{N}$ that are still an admissible $N$-tuple of potentials such that $\varphi_{i} \leq \tilde{\varphi}_{i}$ and

$$
\inf \tilde{\varphi}_{i}-\sup \tilde{\varphi}_{i} \leq-\left(\begin{array}{c}
N \\
2
\end{array}\right)\|c\|_{\infty} .
$$

Now if $\varphi_{1}, \ldots, \varphi_{N}$ were maximizing potentials (their existence is proven, for example, in [22, Proposition 2.3 and Theorem 2.21]), we would have that $\tilde{\varphi}_{i}$ are also maximizing potentials. We can then assume that $\sup \tilde{\varphi}_{i}=t>0$ is independent of $i$, implying also that $t \leq \frac{N-1}{2}\|c\|_{\infty}$.

Then we can consider $\varphi(x)=\frac{1}{N} \sum_{i=1}^{N} \tilde{\varphi}_{i}(x)$, which will be a maximizing potential with the required property (also in the Lipschitz and concave hypothesis).

Copyright $@$ by SIAM. Unauthorized reproduction of this article is prohibited. 
Proof of Proposition 1.5. We can just apply Lemma 5.1 and then Theorem 5.2." We are now ready to prove one of the main results.

Proof of Theorem 1.2. We deal separately with each individual point.

(i) Let $\rho, \mu \in \mathscr{K}_{r, \delta}$ and $h:=h(r, \delta)$ be given by (5.3). We can assume $\mathscr{C}(\rho) \geq \mathscr{C}(\mu)$; let us consider then a potential $\varphi_{\mu}$ relative to $\mu$ given by Theorem 1.5. In particular, we have

$$
\mathscr{C}(\mu)-\mathscr{C}(\rho) \leq \int \varphi_{\mu} \mathrm{d}(\mu-\rho) \leq\left\|\varphi_{\mu}\right\|_{\infty} \cdot\|\mu-\rho\|_{\mathrm{TV}} \leq\left(\begin{array}{c}
N \\
2
\end{array}\right) h \cdot\|\mu-\rho\|_{\mathrm{TV}} .
$$

(ii) If $\rho_{n} \rightarrow \rho$, they all satisfy $\kappa\left(\rho_{n}, r^{\prime}\right)<1 / N-\delta$ for some $r^{\prime}>0$, thanks to Lemma 2.3. But then by Lemma 5.1 there exists $h>0$ such that $\mathscr{C}^{h}$ coincides with $\mathscr{C}$ on the whole sequence. Thanks to the fact that $c^{h}$ is continuous and bounded, the corresponding functional $\mathscr{C}^{h}$ is weakly continuous, and so we reach the conclusion.

(iii) Let $\rho, \mu \in \mathscr{K}_{r, \delta}$ and $h:=h(r, \delta)$ be given by (5.3). We can assume $\mathscr{C}(\rho) \geq \mathscr{C}(\mu)$; let us consider then a potential $\varphi_{\mu}$ relative to $\mu$ given by Proposition 1.5. In particular, we have that $\frac{\varphi_{\mu}}{(N-1)\left\|c^{h}\right\|_{\text {Lip }}}$ is 1-Lipschitz; by the duality formula for $W_{1}$ we then have

$$
\begin{aligned}
\mathscr{C}(\mu)-\mathscr{C}(\rho) & \leq \int \varphi_{\mu} \mathrm{d}(\mu-\rho)=(N-1)\left\|c^{h}\right\|_{\text {Lip }} \int \frac{\varphi_{\mu}}{(N-1)\left\|c^{h}\right\|_{\text {Lip }}} \mathrm{d}(\mu-\rho) \\
& \leq(N-1)\left\|c^{h}\right\|_{\text {Lip }} \cdot W_{1}(\mu, \rho) .
\end{aligned}
$$

Remark 5.3. It is not necessary for the cost to be Lipschitz outside each $D_{\alpha}$; it would be enough to have it be Lipschitz where the plans are supported; see, for example, subsection 6.1 .

6. The case of Coulomb cost. In this section we will resume the main results of the paper in the case of $X=\mathbb{R}^{d}$ and $c(x, y)=\frac{1}{|x-y|}$. First we can take $m(r)=$ $M(r)=r^{-1}$. In particular, the assumptions (1.1) and (1.2) are satisfied. In what follows, the gauge function $g$ will also be useful for summarizing some estimates:

$$
g(\delta)= \begin{cases}2(N-1) & \text { if } \delta<\frac{1}{2(N-1)} \\ \frac{N^{2}(N-1)}{2(1-N \delta)} & \text { if } \frac{1}{2(N-1)} \leq \delta<\frac{1}{N} \\ +\infty & \text { otherwise }\end{cases}
$$

In this section $\mathscr{C}$ will be the cost introduced in (1.4) with the choice $c(x, y)=$ $\frac{1}{|x-y|}$. Whenever used, we will have that $\rho \in \mathscr{P}\left(\mathbb{R}^{d}\right), r>0, \delta \in(0,1 / N)$, and $\mathscr{K}_{r, \delta}$ will be defined as in (1.8). Moreover, $\pi$ will denote any optimal plan for problem (1.4) relative to $\rho$. All the results of the paper concerning the Coulomb case are collected in Table 1.

A couple of remarks are in order: the finiteness conditions of Theorem 1.1 and the bound in Remark 4.2 are already clear. As for the diagonal bounds, when we consider the Coulomb cost, of course we have $D_{r}=D^{1 / r}$, and so we will work directly with the more geometric $D_{r}$. The results Theorem 1.3(i) and (ii), which involve an estimate of the cost, are unified thanks to the gauge function $g$ defined in (6.1).

It is worthwhile to add here a statement that goes in the opposite direction.

Lemma 6.1. Let $\rho \in \mathscr{P}(X)$ and $\pi \in \Pi_{N}(\rho)$. Then if $\kappa(\rho, r)>\frac{1}{N}$, we have $\pi\left(D_{2 r}\right) \neq 0$. 
Proof. The same reasoning behind the proof of Theorem 1.1(ii) allows us to prove the following: whenever $\rho(A)>\frac{1}{N}$, we have $\pi\left(\bigcup_{i \neq j}\left\{x_{i}, x_{j} \in A\right\}\right) \neq 0$. But then, by hypothesis there exists $x \in X$ such that $\rho(B(x, r))>\frac{1}{N}$, and in particular there exists $\left(x_{1}, \ldots, x_{N}\right)$ in the support of $\pi$ such that $x_{i}, x_{j} \in B(x, r)$ and $i \neq j$; this means that $\left|x_{i}-x_{j}\right|<2 r$, and so we can conclude that $\pi\left(D_{2 r}\right) \neq 0$.

The estimates for the potential are clear once we observe that for the Coulomb cost $c(x, y)=\frac{1}{|x-y|}$ we have the following:

- $c^{h}$ is $h^{2}$-Lipschitz.

- $c^{h}$ is $-\frac{3}{2} h^{3}$-concave.

Then it is sufficient to combine Theorem 5.2 with Lemma 5.1 to obtain the estimate for the potential. In Table 1 we also present two sharper results for the regularity of the potential in the case when $\operatorname{supp} \rho=\mathbb{R}^{d}$; these will be proved in subsection 6.1.

Finally, the explicit Lipschitz constants in Theorem 1.2 are found using the explicit estimates for the potentials.

6.1. Sharper estimates for the potentials. Here we discuss a different approach for the estimates of the potentials; this approach is tailored for $\mathbb{R}^{d}$ and the Coulomb cost in the case where we also have $\operatorname{supp} \rho=\mathbb{R}^{d}$. First, in the proof of Theorem 1.3, we can prove the sharper estimate

$$
\sum_{i=2}^{N-1} \frac{1}{\left|x_{1}-x_{i}\right|} \leq \frac{g(\delta)}{r} \quad \text { for } \pi \text {-almost every }\left(x_{1}, x_{2}, \ldots, x_{N}\right) .
$$

In particular, then, when we consider the optimal potential $\varphi$ (which is unique thanks to the assumption $\operatorname{supp} \rho=\mathbb{R}^{d}$ ), since we have

$$
\begin{gathered}
\varphi\left(x_{1}\right)+\cdots+\varphi\left(x_{N}\right) \leq c\left(x_{1}, \ldots, x_{N}\right) \quad \forall x_{1}, \ldots, x_{N} \in \mathbb{R}^{d}, \\
\varphi\left(x_{1}\right)+\cdots+\varphi\left(x_{N}\right)=c\left(x_{1}, \ldots, x_{N}\right) \quad \text { on } \operatorname{supp} \pi,
\end{gathered}
$$

letting $A(x)=\left\{\left(x_{2}, x_{3}, \ldots, x_{N}\right): \sum_{i=2}^{N-1} \frac{1}{\left|x-x_{i}\right|} \leq \frac{g(\delta)}{r}\right\}$ we can say

$$
\begin{aligned}
\varphi(x) & =\min \left\{c\left(x, \ldots, x_{N}\right)-\left(\varphi\left(x_{2}\right)+\cdots+\varphi\left(x_{N}\right)\right):\left(x, x_{2}, \ldots, x_{N}\right) \in \operatorname{supp}(\pi)\right\} \\
& =\min \left\{c\left(x, \ldots, x_{N}\right)-\left(\varphi\left(x_{2}\right)+\cdots+\varphi\left(x_{N}\right)\right):\left(x_{2}, \ldots, x_{N}\right) \in A(x)\right\} .
\end{aligned}
$$

Now it is sufficient to study the regularity knowing this representation. In general, we could think of something like

$$
\varphi(x)=\min \left\{f_{i}(x): i \in I\right\}=\min \left\{f_{i}(x): i \in I(x)\right\} .
$$

For representations like this the idea for controlling the regularity is to consider $I^{\varepsilon}(x)$ such that $I(y) \subset I^{\varepsilon}(x)$ for every $y$ in a neighborhood of $x$; then we estimate the Lipschitz constant and the concavity of $\varphi$ pointwise and then let $\varepsilon \rightarrow 0$. If the functions $f_{i}$ are uniformly $C^{3}$ around $x$ for $i \in I^{\varepsilon}(x)$, we could say

$$
|\nabla \varphi|(x) \leq \lim _{\varepsilon \rightarrow 0} \sup _{i \in I^{\varepsilon}(x)}\left|\nabla f_{i}\right|(x), \quad D^{2} \varphi(x) \leq \lim _{\varepsilon \rightarrow 0} \sup _{i \in I^{\varepsilon}(x)} D^{2} f_{i}(x) .
$$

In our case, for example, we can say

$$
|\nabla \varphi|(x) \leq \lim _{\varepsilon \rightarrow 0} \sup \left\{\left|\nabla_{x} c\left(x, x_{2}, \ldots, x_{N}\right)\right|: \sum_{i=2}^{N-1} \frac{1}{\left|x-x_{i}\right|} \leq \frac{g(\delta)+\varepsilon}{r}\right\} \leq \frac{g(\delta)^{2}}{r^{2}}
$$




$$
D^{2} \varphi(x) \leq \lim _{\varepsilon \rightarrow 0} \sup \left\{D_{x}^{2} c\left(x, x_{2}, \ldots, x_{N}\right): \sum_{i=2}^{N-1} \frac{1}{\left|x-x_{i}\right|} \leq \frac{g(\delta)+\varepsilon}{r}\right\} \leq \frac{3 g(\delta)^{3}}{2 r^{3}} \mathrm{Id},
$$

where we used $\left|\nabla_{x} c\left(x, x_{2}, \ldots, x_{N}\right)\right| \leq \sum_{i} \frac{1}{\left|x-x_{i}\right|^{2}} \leq\left(\sum_{i} \frac{1}{\left|x-x_{i}\right|}\right)^{2}$ and similar reasoning for the estimate of $D^{2} \varphi$.

TABLE 1

This is a summary of results for the Coulomb cost. All the results are derived directly from the theorems in the paper, written down directly in the case when $c$ is the Coulomb cost. The only sharper result is for the potential estimates in the case when $\operatorname{supp} \rho$ is the whole $\mathbb{R}^{d}$; those estimates are proved in section 6.1 .

\begin{tabular}{|c|c|}
\hline Cost finiteness & $\begin{array}{l}\text { If } a(\rho)<\frac{1}{N} \text {, then } \mathscr{C}(\rho)<\infty \text {. } \\
\text { If } a(\rho)>\frac{1}{N} \text {, then } \mathscr{C}(\rho)=\infty \text {. } \\
\text { If } a(\rho)=\frac{1}{N} \text {, then } \mathscr{C}(\rho)<\infty \text { if and only if } \\
\qquad \frac{1}{\left|x-x_{0}\right|} \in L_{l o c}^{1}(\rho) \quad \forall x_{0} \in \mathbb{R}^{d} \text { s.t. } \rho\left(\left\{x_{0}\right\}\right)=\frac{1}{N}\end{array}$ \\
\hline Cost estimate & If $\kappa(\rho, r) \leq \frac{1}{N}$, then $\mathscr{C}(\rho) \leq\left(\begin{array}{c}N \\
2\end{array}\right) \frac{1}{r}$ \\
\hline Diagonal estimate & If $\rho \in \mathscr{K}_{r, \delta}$ and $r^{\prime}<\frac{r}{g(\delta)}$, then $\pi\left(D_{r^{\prime}}\right)=0$ \\
\hline Potential estimates & $\begin{array}{l}\text { If } \rho \in \mathscr{K}_{r, \delta} \text {, there exists a potential } \varphi \text { such that } \\
\left.\qquad \begin{array}{c}N \\
2\end{array}\right) \frac{g(\delta)}{r} \leq \varphi(x) \leq \frac{g(\delta) \cdot(N-1)}{2 r}, \\
\qquad \varphi \|_{\text {Lip }} \leq \frac{(N-1) \cdot g(\delta)^{2}}{r^{2}}, \\
\qquad D^{2} \varphi \leq \frac{3(N-1) \cdot g(\delta)^{3}}{2 r^{3}} \text { Id. } \\
\text { If, moreover, we have } \operatorname{supp} \rho=\mathbb{R}^{d}, \text { we can also assume } \\
\qquad\|\varphi\|_{\text {Lip }} \leq \frac{g(\delta)^{2}}{r^{2}}, \quad D^{2} \varphi \leq \frac{3 g(\delta)^{3}}{2 r^{3}} \text { Id } .\end{array}$ \\
\hline Continuity for $\mathscr{C}$ & $\begin{array}{l}\text { For every } \rho_{1}, \rho_{2} \in \mathscr{K}_{r, \delta} \text { we have } \\
\qquad \begin{array}{l}\left|\mathscr{C}\left(\rho_{1}\right)-\mathscr{C}\left(\rho_{2}\right)\right| \leq \frac{(N-1) \cdot g(\delta)^{2}}{r^{2}} \cdot W_{1}\left(\rho_{1}, \rho_{2}\right) \\
\left|\mathscr{C}\left(\rho_{1}\right)-\mathscr{C}\left(\rho_{2}\right)\right| \leq \frac{N(N-1) \cdot g(\delta)}{2 r} \cdot\left\|\rho_{1}-\rho_{2}\right\|_{\mathrm{TV}} \cdot\end{array}\end{array}$ \\
\hline
\end{tabular}

Copyright $@$ by SIAM. Unauthorized reproduction of this article is prohibited. 
Acknowledgments. Part of this work was done while Simone Di Marino was in Paris as an FSMP visitor researcher in INRIA, Équipe MokaPlan, where he had the opportunity to invite Federico Stra. They want to thank the entire team for their warm hospitality, in particular J.D. Benamou, G. Carlier, and L. Nenna. Simone Di Marino also wants to thank F. Santambrogio and Q. Mérigot for useful discussion of the link between the marriage theorem and optimal transport theory, and D. Lombardo for the reference on the Hajnal-Szemerédi theorem.

\section{REFERENCES}

[1] L. Ambrosio, N. Gigli, And G. Savaré, Gradient Flows in Metric Spaces and the Wasserstein Spaces of Probability Measures, Lectures in Mathematics, ETH Zurich, Birkhäuser, Berlin, 2005.

[2] U. Bindini, Marginals with Finite Repulsive Cost, preprint, https://arxiv.org/abs/1702.06301, 2017.

[3] U. Bindini and L. De Pascale, Optimal transport with Coulomb cost and the semiclassical limit of density functional theory, J. Éc. Polytech. Math., 4 (2017), pp. 909-934, https: //doi.org/10.5802/jep.59.

[4] G. Bouchitté, G. Buttazzo, T. Champion, and L. D. Pascale, Dissociating Limit in Density Functional Theory with Coulomb Optimal Transport Cost, preprint, https://arxiv.org/ abs/1811.12085, 2018.

[5] G. Buttazzo, T. Champion, and L. De Pascale, Continuity and estimates for multimarginal optimal transportation problems with singular costs, Appl. Math. Optim., 78 (2018), pp. 185-200, https://doi.org/10.1007/s00245-017-9403-7.

[6] G. Buttazzo, L. De Pascale, and P. Gori-Giorgi, Optimal-transport formulation of electronic density-functional theory, Phys. Rev. A, 85 (2012), 062502, https://doi.org/10.1103/ PhysRevA.85.062502.

[7] M. Colombo, L. De Pascale, and S. Di Marino, Multimarginal optimal transport maps for one-dimensional repulsive costs, Canad. J. Math., 67 (2015), pp. 350-368, https://doi.org/ 10.4153/CJM-2014-011-x.

[8] M. Colombo and S. Di Marino, Equality between Monge and Kantorovich multimarginal problems with Coulomb cost, Ann. Mat. Pura Appl. (4), 194 (2015), pp. 307-320, https: //doi.org/10.1007/s10231-013-0376-0.

[9] M. Colombo and F. Stra, Counterexamples in multimarginal optimal transport with Coulomb cost and spherically symmetric data, Math. Models Methods Appl. Sci., 26 (2016), pp. 1025-1049, https://doi.org/10.1142/S021820251650024X.

[10] C. Cotar, G. Friesecke, and C. Klüppelberg, Density functional theory and optimal transportation with Coulomb cost, Comm. Pure Appl. Math., 66 (2013), pp. 548-599, https://doi.org/10.1002/cpa.21437.

[11] C. Cotar, G. Friesecke, and B. Pass, Infinite-body optimal transport with Coulomb cost, Calc. Var. Partial Differential Equations, 54 (2015), pp. 717-742, https://doi.org/10.1007/ s00526-014-0803-0.

[12] C. Cotar And M. Petrache, Next-order asymptotic expansion for $N$-marginal optimal transport with Coulomb and Riesz costs, Adv. Math., 344 (2019), pp. 137-233, https: //doi.org/10.1016/j.aim.2018.12.008.

[13] L. De Pascale, Optimal transport with Coulomb cost. Approximation and duality, ESAIM Math. Model. Numer. Anal., 49 (2015), pp. 1643-1657, https://doi.org/10.1051/m2an/ 2015035.

[14] C. Dellacherie and P.-A. Meyer, Probabilities and Potential, North-Holland Math. Stud. 29, North-Holland, Amsterdam, New York, 1978.

[15] S. Di Marino, A. Gerolin, And L. Nenna, Optimal transportation theory with repulsive costs, in Topological Optimization and Optimal Transport in the Applied Sciences, De Gruyter, Berlin, 2017, pp. 204-256.

[16] W. Gangbo and A. ŚwiȨch, Optimal maps for the multidimensional Monge-Kantorovich problem, Comm. Pure Appl. Math., 51 (1998), pp. 23-45, https://doi.org/10.1002/(SICI) 1097-0312(199801)51:1<23::AID-CPA2〉3.0.CO;2-H.

[17] A. Gerolin, A. Kausamo, and T. Rajala, Duality theory for multi-marginal optimal transport with repulsive costs in metric spaces, ESAIM Control Optim. Calc. Var., to appear; preprint, https://arxiv.org/abs/1805.00880, 2018.

Copyright $@$ by SIAM. Unauthorized reproduction of this article is prohibited. 
[18] A. Gerolin, A. Kausamo, and T. Rajala, Non-Existence of Optimal Transport Maps for the Multi-Marginal Repulsive Harmonic Cost, preprint, https://arxiv.org/abs/1805.00417, 2018.

[19] N. Ghoussoub And A. Moameni, A self-dual polar factorization for vector fields, Comm. Pure Appl. Math., 66 (2013), pp. 905-933, https://doi.org/10.1002/cpa.21430.

[20] P. Gori-Giorgi, M. Seidl, and G. Vignale, Density-functional theory for strongly interacting electrons, Phys. Rev. Lett., 103 (2009), 166402, https://doi.org/10.1103/PhysRevLett.103. 166402.

[21] A. Hajnal and E. Szemerédi, Proof of a conjecture of P. Erdös, in Combinatorial Theory and Its Applications, II, North-Holland, Amsterdam, 1970, pp. 601-623.

[22] H. G. Kellener, Duality theorems for marginal problems, Z. Wahrsch. Verw. Gebiete, 67 (1984), pp. 399-432, https://doi.org/10.1007/BF00532047.

[23] H. A. Kierstead and A. V. Kostochka, A short proof of the Hajnal-Szemerédi theorem on equitable colouring, Combin. Probab. Comput., 17 (2008), pp. 265-270, https://doi.org/ 10.1017/S0963548307008619.

[24] Y.-H. KIM AND B. PASS, A general condition for Monge solutions in the multi-marginal optimal transport problem, SIAM J. Math. Anal., 46 (2014), pp. 1538-1550, https://doi.org/10. $1137 / 130930443$.

[25] M. Lewin, E. H. Lieb, and R. Seiringer, Statistical mechanics of the uniform electron gas, J. Éc. Polytech. Math., 5 (2018), pp. 79-116, https://doi.org/10.5802/jep.64.

[26] E. Paolini and E. Stepanov, Decomposition of acyclic normal currents in a metric space, J. Funct. Anal., 263 (2012), pp. 3358-3390, https://doi.org/10.1016/j.jfa.2012.08.009.

[27] B. PAss, On the local structure of optimal measures in the multi-marginal optimal transportation problem, Calc. Var. Partial Differential Equations, 43 (2012), pp. 529-536, https://doi.org/10.1007/s00526-011-0421-z.

[28] M. Petrache, Decorrelation as an Avatar of Convexity, preprint, https://arxiv.org/abs/1507. $00782,2015$.

[29] F. Santambrogio, Optimal Transport for Applied Mathematicians, Birkhäuser, Cham, 2015, https://doi.org/10.1007/978-3-319-20828-2.

[30] M. Seidl, S. Di Marino, A. Gerolin, L. Nenna, K. Giesbertz, and P. Gori-Giorgi, The Strictly-Correlated Electron Functional for Spherically Symmetric Systems Revisited, preprint, https://arxiv.org/abs/1702.05022, 2017.

[31] C. Villani, Topics in Optimal Transportation, Grad. Stud. Math. 58, AMS, Providence, RI, 2003, https://doi.org/10.1090/gsm/058.

Copyright $@$ by SIAM. Unauthorized reproduction of this article is prohibited. 